\title{
Eigenvalue Problems of Quasilinear Elliptic Systems on $\mathbb{R}^{n}$
}

Li Gongbao

\section{Abstract}

In this paper, we get the existence results of the nontrivial weak solution $(\lambda, u)$ of the following eigenvalue problem of quasilinear elliptic systems

$$
-D_{\alpha}\left(a_{\alpha \beta}(x, u) D_{\beta} u^{i}\right)+\frac{1}{2} D_{u^{i}} a_{\alpha \beta}(x, u) D_{\alpha} u^{j} D_{\beta} u^{j}+h(x) u^{i}=\lambda|u|^{p-2} u^{i},
$$

for $x \in \mathbb{R}^{n}, 1 \leqslant i \leqslant N$ and

$$
u=\left(u^{1}, u^{2}, \ldots, u^{N}\right) \in E=\left\{v=\left(v^{1}, v^{2}, \ldots, v^{N}\right) \mid v^{i} \in H^{1}\left(\mathbb{R}^{n}\right), 1 \leqslant i \leqslant N\right\},
$$

where $a_{\alpha \beta}(x, u)$ satisfy the natural growth conditions. It seems that this kind of problem has never been dealt with before.

\section{Introduction}

We consider eigenvalue problems of the following quasilinear elliptic systems on $\mathbb{R}^{n}$

(1.1) $-D_{\alpha}\left(a_{\alpha \beta}(x, u) D_{\beta} u^{i}\right)+\frac{1}{2} D_{u^{i}} a_{\alpha \beta}(x, u) D_{\alpha} u^{j} D_{\beta} u^{j}+h(x) u^{i}=\lambda|u|^{p-2} u^{i}$ 
for $x \in \mathbb{R}^{n}, 1 \leqslant i \leqslant N$ and

$$
u=\left(u^{1}, u^{2}, \ldots, u^{N}\right) \in E=\left\{v=\left(v^{1}, v^{2}, \ldots, v^{N}\right) \mid v^{i} \in H^{1}\left(\mathbb{R}^{N}\right), 1 \leqslant i \leqslant N\right\}
$$

where $R<p<2 \hat{n} /(\hat{n}-2), \hat{n}=n$ if $n>2,2 \hat{n} /(\hat{n}-2)$ is any positive number lager than 2 if $n \leqslant 2$,

$$
D_{\alpha}=\frac{\partial}{\partial x_{\alpha}}, \quad D_{u^{i}}=\frac{\partial}{\partial u^{i}} \quad(1 \leqslant \alpha \leqslant n, \quad 1 \leqslant i \leqslant N)
$$

and the summation conventions have been used and will be used in the following, i.e. the repeated Greek letters and Latin letters denote the sum from 1 to $n$ and 1 to $N$ respectively.

Problem (1.1) comes from the theory of harmonic mappings. There have been some results of (1.1) in bounded domains ([1], [2]). In [1], the existence of solutions for (1.1) is discussed under the conditions

$$
\begin{gathered}
\mu_{1}|\xi|^{2} \leqslant a_{\alpha \beta}(x, u) \xi_{\alpha} \xi_{\beta} \leqslant \mu_{2}|\xi|^{2} \quad \mu_{1}, \mu_{2}>0 \\
\lim _{u \rightarrow+\infty} u D_{u} a_{\alpha \beta}(x, u)=0
\end{gathered}
$$

for every $(u, \xi) \in \mathbb{R}^{1} \times \mathbb{R}^{n}, \quad x \in \Omega \subset \mathbb{R}^{n}$, where $N=1, p=2 n /(n-2), n>2$ if $n>2$. In [2] the existence theorem is obtained when $N \geqslant 1, h \equiv 0,2<p$ $<2 n /(n-2), n>2$ under the conditions

$$
\left\{\begin{array}{l}
a_{1}|\xi|^{2} \leqslant \sigma(|u|)|\xi|^{2} \leqslant a_{\alpha \beta}(x, u) \xi_{\alpha} \xi_{\beta} \leqslant a_{2} \sigma(|u|)|\xi|^{2} \\
\left|u^{i} D_{u^{i}} a_{\alpha \beta}(x, u)\right| \leqslant C \sigma(|u|) \\
\left|D_{u^{i}} a_{\alpha \beta}(x, u)\right| \leqslant C \sigma(|u|), \quad\left|D_{u^{i}} a_{\alpha \beta}(x, u)\right| \leqslant \eta(|u|) \\
-\frac{u^{i}}{2} D_{u^{i}} a_{\alpha \beta}(x, u) \xi_{\alpha} \xi_{\beta} \leqslant a_{3} a_{\alpha \beta}(x, u) \xi_{\alpha} \xi_{\beta} \quad\left(0<a_{3}<1\right),
\end{array}\right.
$$

for every $(x, u, \xi) \in \Omega \times \mathbb{R}^{N} \times \mathbb{R}^{n}$, where $\sigma(t), \eta(t)$ are nonnegative continuous functions on $[0,+\infty)$ satisfying that for any $c_{1}>1$, there exists $c_{2}$, such that $\sigma\left(c_{1} t\right) \leqslant c_{2} \sigma(t)$ for all $t \geqslant 0$.

However, there have not been any results for (1.1) in the unbounded domain $\mathbb{R}^{n}$. Formally, if the minimum of the functional

$$
I(u)=\int_{\mathbb{R}^{n}}\left[a_{\alpha \beta}(x, u) D_{\alpha} u^{i} D_{\beta} u^{i}+h(x)|u|^{2}\right] d x
$$

over the set $\left\{\left.u \in E\left|\int_{\mathbb{R}^{n}}\right| u\right|^{p} d x=\mu\right\}(\mu>0)$ were achieved by some $u$, there should be a $\lambda \in \mathbb{R}^{1}$ such that $(\lambda, u)$ solves (1.1) in a weak sense. But there are some difficulties in dealing with the functional $I(u)$. Firstly, because of the unboundedness of $\mathbb{R}^{n}$, the Sobolev embedding is not compact and the standard convex-compactness techniques can not be used, at least in a straightforward 
way as in the case of bounded domains, and this makes the problem of the existence of a minimizer more difficult. Secondly, the space where $I$ is differentiable is $L_{\infty} \cap E$ (see [3]), so even if we had found a minimizer $u \in E$ of $I$, we could not conclude the existence of $(\lambda, u) \mathbb{R}^{1} \times E$ solving (1.1), unless we had known that $u \in L_{\infty}$. But, usually, the fact that $\|u\|_{\infty}$ is finite is obtained because $u$ satisfies the related Euler equation which in turn is a consequence of the differentiability of $I$ at $u$. This makes the problem complicated.

To overcome the first difficulty, we use the concentration compactness principle, recently developed by P. L. Lions ([4], [5]), when treating the constrained variational problems in unbounded domains. To overcome the second difficulty, we first show that, for any minimizer $u$ of $I$ and some $\varphi \in E$,

$$
\left.\frac{d}{d t} I(u+t \varphi)\right|_{t=0}=0
$$

i.e. the Euler equation related to the functional $I$ holds in a weak sense for $u$ over special test functions in $E$. We then use the Nash-Moser methods to show that $\|u\|_{\infty}$ is finite and finally we get the existence of a nontrivial solution $(\lambda, u)$ of (1.1).

\section{Main Results}

In this section, we present the main results of this paper. First of all, we give some notations and conditions.

Let $H^{1}\left(\mathbb{R}^{n}\right)$ be the usual Sobolev space, $N \geqslant 1$ be a natural number and $E=\left\{u=\left(u^{1}, u^{2}, \ldots, u^{N}\right) \mid u^{i} \in H^{1}\left(\mathbb{R}^{n}\right), 1 \leqslant i \leqslant N\right\}$. The scalar product of $u, v \in E$ is defined by

$$
\langle u, v\rangle=\int_{\mathbb{R}^{n}}\left[D_{\alpha} u^{i} D_{\alpha} v^{i}+u^{i} v^{i}\right] d x
$$

and $(E,<,>)$ is a Hilbert space, the norm of $u \in E$ is $\|u\|_{E}=\left(\|\mid D u\|_{2}^{2}+\right.$ $\left.\|u\|_{2}^{2}\right)^{1 / 2}$ where hereafter $\|f\|_{q}$ denotes the $L^{q}\left(\mathbb{R}^{n}\right)$ norm of the function $f$ and $|f|$ denotes the Euclidean norm of the function $f$ (possibly vector valued). For simplicity, we denote $\|u\|_{E}$ by $\|u\|$ for $u \in E$.

The main conditions imposed on (1.1) will be the following

(i) $2<p<2 \hat{n} /(\hat{n}-2)$ where $\hat{n}=n$ if $n>2$; and $2 \hat{n} /(\hat{n}-2)$ is any positive number larger than 2 if $n \leqslant 2$.

(ii) $a_{\alpha \beta}(x, u) \in C^{1}\left(\mathbb{R}^{n} \times \mathbb{R}^{N}\right), a_{\alpha \beta}=a_{\beta \alpha}$ for any $\alpha, \beta$ and $a_{1}>0, a_{2}>1$ such that for any $(x, u, \xi) \in \mathbb{R}^{n} \times \mathbb{R}^{N} \times \mathbb{R}^{n}$

$$
a_{1}|\xi|^{2} \leqslant \sigma(|u|)|\xi|^{2} \leqslant a_{\alpha \beta}(x, u) \xi_{\alpha} \xi_{\beta} \leqslant a_{2} \sigma(|u|)|\xi|^{2}
$$


holds, where $\sigma(t)$ is a nonnegative nondecreasing continuous function on $[0,+\infty)$ satisfying: for any $l>1$, there exists $C_{l}>0$, such that

$$
\sigma(l t) \leqslant C_{l} \sigma(t), \quad \text { for all } t \geqslant 0
$$

and $C_{l}$ are bounded whenever $l$ are bounded. Moreover, there is a constant $C>0$ with

$$
\sigma(t) \leqslant C\left(1+|t|^{q}\right)
$$

where $0 \leqslant q \leqslant 4 /(n-2)$ if $n>2$ and $0 \leqslant q$ if $n \leqslant 2$.

(iii) $a_{\alpha \beta}(x, u) \rightarrow \bar{a}_{\alpha \beta}(u)$ as $|x| \rightarrow+\infty$ uniformly for $u$ bounded.

(iv) There exists, $s \geqslant 0, s<p-2$ such that

$$
\begin{gathered}
a_{\alpha \beta}(x, \lambda u) \xi_{\alpha} \xi_{\beta} \leqslant \lambda^{s} a_{\alpha \beta}(x, u) \xi_{\alpha} \xi_{\beta} \\
a_{\alpha \beta}(x, u) \xi_{\alpha} \xi_{\beta} \leqslant \bar{a}_{\alpha \beta}(u) \xi_{\alpha} \xi_{\beta}
\end{gathered}
$$

for any $(x, u, \xi) \in \mathbb{R}^{n} \times \mathbb{R}^{N} \times \mathbb{R}^{n}$, where $p$ is given in (i) and $\bar{a}_{\alpha \beta}$ are defined in (iii), and $\lambda>1$ is arbitrary.

(v) $h \in C\left(\mathbb{R}^{n}\right)$ and there are $\bar{h}, c>0$ such that $h(x) \geqslant c, h(x) \leqslant \bar{h}$ for any $x \in \mathbb{R}^{n}$ and $\lim _{|x| \rightarrow \infty} h(x)=\bar{h}$.

(vi) There is a constant $c>0$ such that

$$
\begin{aligned}
\left|u^{i} D_{u^{i}} a_{\alpha \beta}(x, u)\right| & \leqslant c \sigma(|u|) \\
\left|D_{u^{i}} a_{\alpha \beta}(x, u)\right| & \leqslant c \eta(|u|)
\end{aligned}
$$

for any $(x, u) \in \mathbb{R}^{n} \times \mathbb{R}^{N}$, where $\eta(t)$ is a nonnegative nondecreasing continuous function on $[0,+\infty)$ and $\sigma(t)$ is given in (ii).

(vii) There is a constant $a_{3}$ with $0<a_{3}<1$ such that

$$
-\frac{1}{2} u^{i} D_{u^{i}} a_{\alpha \beta}(x, u) \xi_{\alpha} \xi_{\beta} \leqslant a_{3} a_{\alpha \beta}(x, u) \xi_{\alpha} \xi_{\beta}
$$

for any $(x, u, \xi) \in \mathbb{R}^{n} \times \mathbb{R}^{N} \times \mathbb{R}^{n}$.

Remark 2.1. If $a_{\alpha \beta}(x, u), h(x)$ satisfy (i)-(vii), then $\bar{a}_{\alpha \beta}(u), \bar{h}$ satisfy (i)-(vii).

If $a_{\alpha \beta}(x, u), h(x)$ satisfy (i)-(v), we set, for any $u \in E$

$$
\begin{aligned}
I(u) & =\int_{\mathbb{R}^{n}}\left(a_{\alpha \beta}(x, u) D_{\alpha} u^{i} D_{\beta} u^{i}+h(x)|u|^{2}\right) d x \\
I^{\infty}(u) & =\int_{\mathbb{R}^{n}}\left(\bar{a}_{\alpha \beta}(u) D_{\alpha} u^{i} D_{\beta} u^{i}+\bar{h}|u|^{2}\right) d x
\end{aligned}
$$

For any $\lambda>0$, we set

$$
\begin{aligned}
I_{\lambda} & =\inf \left\{\left.I(u)\left|u \in E, \int_{\mathbb{R}^{n}}\right| u\right|^{p} d x=\lambda\right\} \\
I_{\lambda}^{\infty} & =\inf \left\{\left.I^{\infty}(u)\left|u \in E, \int_{\mathbb{R}^{n}}\right| u\right|^{p} d x=\lambda\right\}
\end{aligned}
$$


It is clear that

$$
\begin{gathered}
I_{\lambda}=\inf \left\{\left.I\left(\lambda^{1 / p} u\right)\left|u \in E, \int_{\mathbb{R}^{n}}\right| u\right|^{p} d x=1\right\} \\
I_{\lambda}^{\infty}=\inf \left\{\left.I^{\infty}\left(\lambda^{1 / p} u\right)\left|u \in E, \int_{\mathbb{R}^{n}}\right| u\right|^{p} d x=1\right\}
\end{gathered}
$$

The pair $(\lambda, u) \in \mathbb{R}^{1} \times E$ will be called a weak solution of (1.1) if

$$
\begin{aligned}
\int_{\mathbb{R}^{n}}\left[a_{\alpha \beta}(x, u) D_{\alpha} u^{i} D_{\beta} \varphi^{i}+\varphi^{i} D_{u^{j}} a_{\alpha \beta}(x, u) D_{\alpha} u^{i} D_{\beta} u^{i}+h(x) u^{i} \varphi^{i}\right] d x & \\
& =\lambda \int_{\mathbb{R}^{n}}|u|^{p-2} u^{i} \varphi^{i} d x
\end{aligned}
$$

for any $\varphi \in L_{\infty} \cap E$.

It is evident that $u=0$ is a trivial solution of (1.1) for any $\lambda$.

The main results of this paper are the following

Theorem 2.1. Suppose that (i)-(vi) hold, then for any $\lambda>0, I_{\lambda}^{\infty}$ is achieved by some $u \in E$.

Theorem 2.2. Suppose that (i)-(vi) hold, then there is a $\lambda_{0}>0$ such that $I_{\lambda_{0}}$ is achieved by some $u \in E$. Moreover, if $I_{\lambda}<I_{\lambda}^{\infty}$ for any $\lambda>0$, then $I_{\lambda}$ is achieved by some $u \in E$ for any $\lambda>0$.

Theorem 2.3. Suppose that (i)-(vii) hold, then (1.1) posesses at least a nontrivial weak solution $(\lambda, u) \in \mathbb{R}^{1} \times E$ and $\|u\|_{\infty}<\infty$.

Remark 2.2. By (iv)-(v), it is trivial that $I_{\lambda} \leqslant I_{\lambda}^{\infty}$, and by Theorem 2.1, $I_{\lambda}<I_{\lambda}^{\infty}$ (for all $\lambda>0$ ) if

$$
\int_{\mathbb{R}^{n}}\left[a_{\alpha \beta}(x, u) D_{\alpha} u^{i} D_{\beta} u^{i}+h(x)|u|^{2}\right] d x<\int_{\mathbb{R}^{n}}\left[\bar{a}_{\alpha \beta}(u) D_{\alpha} u^{i} D_{\beta} u^{i}+\bar{h}|u|^{2}\right] d x
$$

holds for $u \in E, \int_{\mathbb{R}^{n}}|u|^{p} d x=\lambda$ with $I^{\infty}(u)=I_{\lambda}{ }^{\infty}<\infty$. (2.15) is valid, for instance, when $h(x)<\bar{h}$ for any $x \in \mathbb{R}^{n}$, or $a_{\alpha \beta}(x, u) \xi_{\alpha} \xi_{\beta}<\bar{a}_{\alpha \beta}(u) \xi_{\alpha} \xi_{\beta}$ for any $(x, u, \xi) \in \mathbb{R}^{n} \times\left(\mathbb{R}^{N}-\{0\}\right) \times\left(\mathbb{R}^{n}-\{0\}\right)$.

EXAmPle 2.1. In (1.1), if $n=3, p=5, h(x)$ satisfies (v), and

$$
a_{\alpha \beta}(x, u)=\left(1+|u|^{2}\right) b_{\alpha \beta}(x) \quad\left(\text { or, } a_{\alpha \beta}(x, u)=b_{\alpha \beta}(x) /\left(1+|u|^{2}\right)\right)
$$

where $b_{\alpha \beta}(x) \in C^{1}\left(\mathbb{R}^{n}\right)$ and $b_{\alpha \beta}=b_{\beta \alpha}(1 \leqslant \alpha, \beta \leqslant n)$ satisfy

$$
0<\lambda|\xi|^{2} \leqslant b_{\alpha \beta}(x) \xi_{\alpha} \xi_{\beta} \leqslant M|\xi|^{2}
$$

for any $(x, \xi) \in \mathbb{R}^{n} \times \mathbb{R}^{n}$ where $\lambda, M>0$ are constants, and $\lim _{|x| \rightarrow \infty} b_{\alpha \beta}(x)$ $=\bar{b}_{\alpha \beta}$, then, it is easy to see that $a_{\alpha \beta}(x, u), h(x)$ satisfy conditions (i)-(vii), and 
thus we conclude that (1.1) possesses at least a nontrivial weak solution by using Theorem 2.3.

The above is only a simple example, the theorems in this section are applicable to many other cases.

\section{Proof of Theorems 2.1 and 2.2}

In this section, we prove Theorem 2.1 and Theorem 2.2. We need some lemmata and we always suppose that conditions (i)-(v) hold in this section.

Lemma 3.1. $I_{\lambda}, I_{\lambda}^{\infty}$ are continuous functions of $\lambda$ on $[0,+\infty)$.

Proof. It is evident that $I_{\lambda}, I_{\lambda}^{\infty}$ are all finite for each $\lambda \geqslant 0$. Let $\lambda_{m} \rightarrow \lambda_{0}$ $\epsilon(0,+\infty)$. We may assume that $\lambda_{m}>0$ for any $m>0$. Given $\epsilon>0$ we have by (2.13), that there are $\left(u_{m}\right) \subset E$ such that $\int_{\mathbb{R}^{n}}\left|u_{m}\right|^{p} d x=1$ and

$$
I\left(\lambda_{m}^{1 / p} u_{m}\right) \leqslant I_{\lambda_{m}}+\epsilon .
$$

We claim that $\left|I_{\lambda_{m}}\right| \leqslant C$ (hereafter $C$ denotes a constant independent of $m$ ). In fact, for fixed $u_{0} \in C_{0}^{\infty} \subset E$ with $\int_{\mathbb{R}^{n}}\left|u_{0}\right|^{p} d x=1$, we have by (2.1), the fact that $\left|\lambda_{m}\right| \leqslant C$ and the continuity of $\sigma(t)$, that

$$
\begin{aligned}
I_{\lambda_{m}} & \leqslant I\left(\lambda_{m}^{1 / p} u_{0}\right)=\lambda_{m}^{2 / p} \int_{\mathbb{R}^{n}}\left[a_{\alpha \beta}\left(x, \lambda_{m}^{1 / p} u_{0}\right) D_{\alpha} u_{0}^{i} D_{\beta} u_{0}^{i}+h(x)\left|u_{0}\right|^{2}\right] d x \\
& \leqslant \lambda_{m}^{2 / p} \int_{\mathbb{R}^{n}} \sigma\left(\left|\lambda_{m}^{1 / p} u_{0}\right|\right)\left|D u_{0}\right|^{2}+\lambda_{m}^{2 / p} \int_{\mathbb{R}^{n}} h(x)\left|u_{0}\right|^{2} d x \leqslant C<+\infty .
\end{aligned}
$$

Hence, by (ii) we get

$$
\int_{\mathbb{R} n}\left[\sigma\left(\lambda_{m}^{1 / p}\left|u_{m}\right|\right)\left|D u_{m}\right|^{2}+h(x)\left|u_{m}\right|^{2}\right] d x \leqslant I_{\lambda_{m}}+\epsilon \leqslant C .
$$

Since $\sigma(t)$ is nondecreasing in $t$, it is trivial that

$$
\int_{\mathbb{R}^{n}}\left[\sigma\left(\lambda_{0}^{1 / p}\left|u_{m}\right|\right)\left|D u_{m}\right|^{2}+h(x)\left|u_{m}\right|^{2}\right] d x \leqslant C
$$

when $\lambda_{m} \geqslant \lambda_{0}$, while if $\lambda_{m}<\lambda_{0}$, we have by (2.2) and the boundedness of $\left(\lambda_{0} / \lambda_{m}\right)^{1 / p}$, that

$$
\begin{aligned}
\int_{\mathbb{R}^{n}}\left[\sigma\left(\lambda_{0}^{1 / p}\left|u_{m}\right|\right)\left|D u_{m}\right|^{2}+h(x)\left|u_{m}\right|^{2}\right] d x \\
\quad=\int_{\mathbb{R}^{n}}\left[\sigma\left(\left(\frac{\lambda_{0}}{\lambda_{m}}\right)^{1 / p} \lambda_{m}^{1 / p}\left|u_{m}\right|\right)\left|D u_{m}\right|^{2}+h(x)\left|u_{m}\right|^{2}\right] d x \\
\leqslant C_{m} \int_{\mathbb{R}^{n}}\left[\sigma\left(\lambda_{m}^{1 / p}\left|u_{m}\right|\right)\left|D u_{m}\right|^{2}+h(x)\left|u_{m}\right|^{2}\right] d x \leqslant C<+\infty .
\end{aligned}
$$


Thus, we always have

$$
\int_{\mathbb{R}^{n}}\left[\sigma\left(\lambda_{0}^{1 / p}\left|u_{m}\right|\right)\left|D u_{m}\right|^{2}+h(x)\left|u_{m}\right|^{2}\right] d x \leqslant C .
$$

It is clear that

$$
\begin{aligned}
I_{\lambda_{m}}+\epsilon & \geqslant I\left(\lambda_{m}^{1 / p} u_{m}\right) \\
& =I\left(\lambda_{m}^{1 / p} u_{m}\right)-I\left(\lambda_{0}^{1 / p} u_{m}\right)+I\left(\lambda_{0}^{1 / p} u_{m}\right) \\
& \geqslant I\left(\lambda_{m}^{1 / p} u_{m}\right)-I\left(\lambda_{0}^{1 / p} u_{m}\right)+I_{\lambda_{0}},
\end{aligned}
$$

but

$$
\begin{aligned}
I\left(\lambda_{m}^{1 / p} u_{m}\right)-I\left(\lambda_{0}^{1 / p} u_{m}\right)= & \lambda_{m}^{2 / p} \int_{\mathbb{R}^{n}}\left[a_{\alpha \beta}\left(x, \lambda_{m}^{1 / p} u_{m}\right)-a_{\alpha \beta}\left(x, \lambda_{0}^{1 / p} u_{m}\right)\right] D_{\alpha} u_{m}^{i} D_{\beta} u_{m}^{i} d x \\
& +\left(\lambda_{m}^{2 / p}-\lambda_{0}^{2 / p}\right) \int_{\mathbb{R}^{n}} a_{\alpha \beta}\left(x, \lambda_{0}^{1 / p} u_{m}\right) D_{\alpha} u_{m}^{i} D_{\beta} u_{m}^{i} d x \\
& +\left(\lambda_{m}^{2 / p}-\lambda_{0}^{2 / p}\right) \int_{\mathbb{R}^{n}} h(x)\left|u_{m}\right|^{2} d x \\
\equiv & I_{m}^{1}+I_{m}^{2}+I_{m}^{3} .
\end{aligned}
$$

It is trivial that $\lim _{m \rightarrow \infty} I_{m}^{3}=0$ and by (2.1) and (3.2) we have that $\lim _{m \rightarrow \infty} I_{m}^{2}$ $=0$. On the other hand, by the mean value theorem, we have

$$
\begin{aligned}
\left|\left[a_{\alpha \beta}\left(x, \lambda_{m}^{1 / p} u_{m}\right)-a_{\alpha \beta}\left(x, \lambda_{0}^{1 / p} u_{m}\right)\right] D_{\alpha} u_{m}^{i} D_{\beta} u_{m}^{i}\right| \\
\quad=\left|\left(\lambda_{m}^{1 / p}-\lambda_{0}^{1 / p}\right) u_{m}^{j} D_{u^{j}} a_{\alpha \beta}\left(x, \xi_{m}(x) u_{m}\right) D_{\alpha} u_{m}^{i} D_{\beta} u_{m}^{i}\right|,
\end{aligned}
$$

where $\xi_{m}(x)$ is between $\lambda_{m}^{1 / p}$ and $\lambda_{0}^{1 / p}$, hence $\left|\xi_{m}(x)\right| \geqslant C>0$. So, by (2.6), (3.1) and (3.2) we have

$$
\begin{aligned}
\left|\int_{\mathbb{R}^{n}} u_{m}^{j} D_{u^{j}} a_{\alpha \beta}\left(x, \xi_{m}(x) u_{m}\right) D_{\alpha} u_{m}^{i} D_{\beta} u_{m}^{i} d x\right| & \leqslant C \int_{\mathbb{R}^{n}} \sigma\left(\xi_{n}(x)\left|u_{m}\right|\right)\left|D u_{m}\right|^{2} d x \\
& \leqslant \max _{0 \leq m} C \int_{\mathbb{R}^{n}} \sigma\left(\lambda_{m}^{1 / p}\left|u_{m}\right|\right)\left|D u_{m}\right|^{2} d x \\
& \leqslant C
\end{aligned}
$$

from which $\lim _{m \rightarrow \infty} I_{m}^{1}=0$ and hence $\liminf _{m \rightarrow \infty} I_{\lambda_{m}}+\epsilon \geqslant I_{\lambda_{0}}$. Thus we have $\liminf { }_{m \rightarrow \infty} I_{\lambda_{m}} \geqslant I_{\lambda_{0}}$ which shows that $I_{\lambda}$ is lower-semi continuous on $(0,+\infty)$. On the other hand, it is trivial to see that $\limsup _{m \rightarrow \infty} I_{\lambda_{m}} \leqslant I_{\lambda_{0}}$, which gives that $I_{\lambda}$ is upper-semi continuous on $(0,+\infty)$. So we see that $I_{\lambda}$ is continuous on $(0,+\infty)$. It is trivial that $I_{\lambda}$ is continuous at $\lambda=0$ and the lemma is proved.

Lemma 3.2. For any $\lambda>0$, we have 


$$
\begin{array}{rlrl}
I_{\lambda} & \leqslant I_{\lambda}^{\infty} & & \\
I_{\lambda}^{\infty}<I_{\alpha}^{\infty}+I_{\lambda-\alpha}^{\infty} & \text { for every } & \alpha \in(0, \lambda) \\
I_{\lambda}<I_{\alpha}+I_{\lambda-\alpha} & \text { for every } & \alpha \in(0, \lambda)
\end{array}
$$

If $I_{\beta}<I_{\beta}^{\infty}$ for any $\beta>0$, then

$$
I_{\lambda}<I_{\alpha}+I_{\lambda-\alpha}^{\infty} \text { for every } \alpha \in[0, \lambda) .
$$

Proof. By (iv) and (v), it is trivial that (3.3) holds. To prove (3.5), we only need to show that

$$
I_{\theta \gamma}<\theta I \quad \text { for every } \quad \gamma \in(0, \lambda), \theta \in\left(1, \frac{\lambda}{\gamma}\right)
$$

(see Lemma II.1 of $[4])$. Given $\gamma \in(0, \lambda), \theta \in\left(1, \frac{\lambda}{\gamma}\right)$, we have by $(2.13)$ and
(2.4), that

$$
\begin{aligned}
I_{\theta \gamma}= & (\theta \gamma)^{2 / p} \inf \left\{\int_{\mathbb{R}^{n}}\left[a_{\alpha \beta}\left(x,(\theta \gamma)^{1 / p} u\right) D_{\alpha} u^{i} D_{\beta} u^{i}+h(x)|u|^{2}\right] d x: u \in E,\right. \\
& \left.\int_{\mathbb{R}^{n}}|u|^{p} d x=1\right\} \\
\leqslant & \theta^{2 / p} \gamma^{2 / p} \theta^{s / p} \inf \left\{\int_{\mathbb{R}^{n}}\left[a_{\alpha \beta}\left(x, \gamma^{1 / p} u\right) D_{\alpha} u^{i} D_{\beta} u^{i}+h(x)|u|^{2}\right] d x: u \in E,\right. \\
& \left.\quad \int_{\mathbb{R}^{n}}|u|^{p} d x=1\right\} \\
= & \theta^{(2+s) / p} I_{\gamma}<\theta I_{\gamma}
\end{aligned}
$$

here we have made use of $I_{\gamma}>0$ (for all $\gamma>0$ ) which can easily be derived from the definition. Thus (3.7) holds and hence (3.5) holds. Similarly, by Remark 2.1 we see that (3.4) holds. By (3.3), (3.5) and $I_{\beta}<I_{\beta}^{\infty}$ (for all $\beta>0$ ), we see that (3.6) holds.

ProOF OF THEOREM 2.1 AND THEOREM 2.2. Let $\left(u_{m}\right) \subset E$ be a minimizing sequence of $I_{\lambda}$ (or $\left.I_{\lambda}^{\infty}\right)$ with

$$
\int_{\mathbb{R}^{n}}\left|u_{m}\right|^{p} d x=\lambda>0
$$

and

$$
I\left(u_{m}\right)<I_{\lambda}+1 / m \quad\left(\text { or } I_{\lambda}^{\infty}\left(u_{m}\right)<I_{\lambda}^{\infty}+1 / m\right) .
$$

Since $I_{\lambda}$ is finite, by (ii) we have

$$
\int_{\mathbb{R}^{n}}\left[\sigma\left(\left|u_{m}\right|\right)\left|D u_{m}\right|^{2}+h(x)\left|u_{m}\right|^{2}\right] d x \leqslant C
$$


(or

$$
\int_{\mathbb{R}^{n}}\left[\sigma\left(\left|u_{m}\right|\right)\left|D u_{m}\right|^{2}+\bar{h}\left|u_{m}\right|^{2}\right] d x \leqslant C
$$

in the case of $I_{\lambda}^{\infty}$ ) and $\left\|u_{m}\right\| \leqslant C$.

By the Sobolev embedding theorem, we may assume the existence of a $u_{0}=\left(u_{0}^{1}, u_{0}^{2}, \ldots, u_{0}^{N}\right) \in E$ such that

$$
\begin{array}{lll}
u_{m} \rightarrow u_{0} & \text { in } E \\
u_{m}^{i}-u_{0}^{i} \text { in } H^{1}\left(\mathbb{R}^{n}\right), & 1 \leqslant i \leqslant N \\
u_{m} \rightarrow u_{0} & \text { a.e. in } \mathbb{R}^{n} \\
u_{m}^{i} \rightarrow u_{0}^{i} \text { in } L_{\mathrm{loc}}^{t}\left(\mathbb{R}^{n}\right), & 2 \leqslant t<\frac{2 \hat{n}}{\hat{n}-2}
\end{array}
$$

where $« \rightarrow$ » designates weak convergence, while $« \rightarrow »$ means strong convergence.

Let

$$
\rho_{m}=a_{\alpha \beta}\left(x, u_{m}\right) D_{\alpha} u_{m}^{i} D_{\beta} u_{m}^{i}+h(x)\left|u_{m}\right|^{2}
$$

(respectively

$$
\rho_{m}=\bar{a}_{\alpha \beta}\left(u_{m}\right) D u_{m}^{i} D u_{m}^{i}+\bar{h}\left|u_{m}\right|^{2}
$$

in the case of $I_{\lambda}^{\infty}$ ), and

$$
\lambda_{m}=\int_{\mathbb{R} n} \rho_{m} d x,
$$

we easily see that $\lambda_{m} \geqslant C>0$. We need the following concentration compactness lemma:

Lemma 3.3. Let $u_{m}, \rho_{m}, \lambda_{m}$ be as above, then there exists a subsequence of $\left(\rho_{m}\right)$, still denoted by $\left(\rho_{m}\right)$, satisfying one of the three following possibilities:

(i) (Compactness) There exists $y_{m} \in \mathbb{R}^{n}$ such that $\rho_{m}\left(x+y_{m}\right)$ is tight, i.e. for every $\epsilon>0$, there exists $R$ such that

$$
\int_{y_{m}+B_{R}} \frac{\rho_{m}(x)}{\lambda_{m}} d x \geqslant 1-\epsilon
$$

where

$$
y_{m}+B_{R}=\left\{x \in \mathbb{R}^{n}:\left|x-y_{m}\right| \leqslant R\right\} .
$$

(ii) (Vanishing) $\lim _{m \rightarrow \infty} \sup _{y \in \mathbb{R}^{n}} \int_{y+B_{R}} \rho_{m}(x) d x=0$ for all $R<+\infty$. 
(iii) (Dichotomy) There exist $\alpha \in(0,1)$ and a positive function $\mu(\epsilon)$, with $\lim _{\alpha \rightarrow 0} \mu(\epsilon)=0$, such that for every $\epsilon>0$ there exist $m_{0} \geqslant 1$ and $u_{m}^{1}, u_{m}^{2} \in E$ with $\left\|u_{m}^{1}\right\|,\left\|u_{m}^{2}\right\| \leqslant C$, so that

$$
\begin{gathered}
\lim _{m \rightarrow \infty} \operatorname{dist}\left(\operatorname{supp} u_{m}^{1}, \operatorname{supp} u_{m}^{2}\right)=+\infty \\
\left\|u_{m}-\left(u_{m}^{1}+u_{m}^{2}\right)\right\|_{2} \leqslant \mu(\epsilon) \\
\left\|u_{m}-\left(u_{m}^{1}+u_{m}^{2}\right)\right\|_{p}<\mu(\epsilon) \\
\left|\frac{I\left(u_{m}^{1}\right)}{\lambda_{m}}-\alpha\right|<\mu(\epsilon) \\
\left|\frac{I\left(u_{m}^{2}\right)}{\lambda_{m}}-(1-\alpha)\right|<\mu(\epsilon) \\
I\left(u_{m}\right) \geqslant I\left(u_{m}^{1}\right)+I\left(u_{m}^{2}\right)-\mu(\epsilon)
\end{gathered}
$$

or, respectively, in the case of $I_{\lambda}^{\infty}$,

$$
\begin{array}{r}
\left|\frac{I^{\infty}\left(u_{m}^{1}\right)}{\lambda_{m}}-\alpha\right|<\mu(\epsilon) \\
\left|\frac{I^{\infty}\left(u_{m}^{2}\right)}{\lambda_{m}}-(1-\alpha)\right|<\mu(\epsilon) \\
I^{\infty}\left(u_{m}\right) \geqslant I^{\infty}\left(u_{m}^{1}\right)+I^{\infty}\left(u_{m}^{2}\right)-\mu(\epsilon) .
\end{array}
$$

Proof. For any $t \geqslant 0$, let

$$
Q_{m}(t)=\sup _{y \in \mathbb{R}^{n}} \int_{y+B_{t}} \frac{\rho_{m}}{\lambda_{m}} d x .
$$

Then $Q_{m}(t)$ is nondecreasing in $t$ and $\left|Q_{m}(t)\right| \leqslant 1$, so by Helly's principle there is a subsequence of $Q_{m}(t)$, still denoted by $Q_{m}(t)$ with $\lim _{m \rightarrow \infty} Q_{m}(t)=Q(t)$ for any $t \geqslant 0$, where $Q(t)$ is a nondecreasing function on $[0,+\infty)$ and $|Q(t)| \leqslant 1$.

Let $\lim _{t \rightarrow \infty} Q(t)=\alpha \in[0,1]$. If $\alpha=0$, then $Q(t) \equiv 0$, hence $\lim _{m \rightarrow \infty} Q_{m}(t)$ $=0$ and (ii) (vaninshing) occurs.

If $\alpha=1$, we can easily show that (i) (compactness) occurs by using the same method as in the proof of Lemma I.1 of [4].

Now, letting $\alpha \in(0,1)$, we want to show that (iii) (dichotomy) occurs.

Given $\epsilon>0$, there exists $R_{0}=R_{0}(\epsilon)>0$ such that

$$
\begin{gathered}
\alpha-\epsilon<Q\left(R_{0}\right)<\alpha+\epsilon \\
\alpha-2 \epsilon<Q\left(2 R_{0}\right)<\alpha+2 \epsilon
\end{gathered}
$$


hence there exists $m_{0}(\epsilon)>0$ with

$$
\begin{gathered}
\alpha-\epsilon<Q_{m}\left(R_{0}\right)<\alpha+\epsilon \\
\alpha-2 \epsilon<Q_{m}\left(2 R_{0}\right)<\alpha+2 \epsilon
\end{gathered}
$$

whenever $m \geqslant m_{0}$.

We may choose $R_{m} \rightarrow+\infty$ such that

$$
Q_{m}\left(2 R_{m}\right)<\alpha+1 / m \text {. }
$$

By the absolute continuity of Lebesgue integrals, there are $\left(z_{m}\right) \subset \mathbb{R}^{n}$ such that

$$
Q_{m}\left(R_{0}\right)=\int_{z_{m}+B_{R_{0}}} \frac{\rho_{m}}{\lambda_{m}} d x
$$

Let $\xi, \varphi \in C_{b}^{\infty}\left(R^{n}\right), 0 \leqslant \xi, \varphi \leqslant 1, \xi \equiv 1$ and $\varphi \equiv 0$ if $|x| \leqslant 1 ; \xi \equiv 0$ and $\varphi \equiv 1$ if $|x| \geqslant 2$ and set $\xi_{m}=\xi\left[\left(x-z_{m}\right) / \tilde{R}\right] / \tilde{R}\left(\tilde{R} \geqslant R_{0}\right.$ is to be determined) $\varphi_{m}=$ $\varphi\left[(x-3 m) / R_{m}\right]$ and $u_{m}^{1}=\xi_{m} u_{m}, u_{m}^{2}=\varphi_{m} u_{m}$. It is trivial that (3.10) holds and that $\left\|u_{m}^{1}\right\|,\left\|u_{m}^{2}\right\| \leqslant C$.

By (3.22) we have

$$
\begin{aligned}
& Q_{m}\left(R_{0}\right)=\frac{1}{\lambda_{m}} \int_{z_{m}+B_{R_{0}}}\left[a_{\alpha \beta}\left(x, u_{m}\right) D_{\alpha} u_{m}^{i} D_{\beta} u_{m}^{i}+h(x)\left|u_{m}\right|^{2}\right] d x \\
& =\frac{1}{\lambda_{m}} \int_{z_{m}+B_{R_{0}}}\left[a_{\alpha \beta}\left(x, \xi_{m} u_{m}\right) D_{\alpha}\left(\xi_{m} u_{m}^{i}\right) D_{\beta}\left(\xi_{m} u_{m}^{i}\right)\right. \\
& \left.+h(x)\left|\xi_{m} u_{m}\right|^{2}\right] d x \\
& =\frac{1}{\lambda_{m}} I\left(u_{m}^{1}\right) \\
& -\frac{1}{\lambda_{m}} \int_{\left|x-z_{m}\right| \geq R_{0}}\left[a_{\alpha \beta}\left(x, u_{m}^{1}\right) D_{\alpha}\left(u_{m}^{1}\right)^{i} D_{\beta}\left(u_{m}^{1}\right)^{i}\right. \\
& \left.+h(x)\left|u_{m}^{1}\right|^{2}\right] d x
\end{aligned}
$$

We want to show that

$$
\frac{1}{\lambda_{m}} \int_{\left|x-z_{m}\right| \geq R_{0}}\left[a_{\alpha \beta}\left(x, u_{m}^{1}\right) D_{\alpha}\left(u_{m}^{1}\right)^{i} D_{\beta}\left(u_{m}^{1}\right)^{i}+h(x)\left|u_{m}^{1}\right|^{2}\right] d x<\mu(\epsilon) .
$$

Since

$$
\frac{1}{\lambda_{m}} \int_{\left|x-z_{m}\right| \geq R_{0}}\left[a_{\alpha \beta}\left(x, u_{m}^{1}\right) D_{\alpha}\left(u_{m}^{1}\right)^{i} D_{\beta}\left(u_{m}^{1}\right)^{i}+h(x)\left|u_{m}^{1}\right|^{2}\right] d x
$$

$$
\begin{gathered}
\leqslant \frac{1}{\lambda_{m}} \int_{R_{0} \leq\left|x-z_{m}\right| \leq 2 \widetilde{R}}\left[a_{\alpha \beta}\left(x, u_{m}^{1}\right)\left(u_{m}^{i} D_{\alpha} \xi_{m}+\xi_{m} D_{\alpha} u_{m}^{i}\right)\left(u_{m}^{i} D_{\beta} \xi_{m}+\xi_{m} D_{\beta} u_{m}^{i}\right)\right. \\
\left.+h(x)\left|u_{m}\right|^{2}\right] d x
\end{gathered}
$$




$$
\begin{aligned}
= & \frac{1}{\lambda_{m}} \int_{R_{0} \leq\left|x-z_{m}\right| \leq 2 R} \xi_{m}^{2} a_{\alpha \beta}\left(x, u_{m}^{1}\right) D_{\alpha} u_{m}^{i} D_{\beta} u_{m}^{i} d x \\
& +\frac{2}{\lambda_{m}} \int_{R_{0} \leq\left|x-z_{m}\right| \leq 2 \widetilde{R}} \xi_{m} u_{m}^{i} a_{\alpha \beta}\left(x, u_{m}^{1}\right) D_{\alpha} \xi_{m} D_{\beta} u_{m}^{i} d x \\
& +\frac{1}{\lambda_{m}} \int_{R_{0} \leq\left|x-z_{m}\right| \leq 2 \widetilde{R}} a_{\alpha \beta}\left(x, u_{m}^{1}\right) D_{\alpha} \xi_{m} D_{\beta} \xi_{m} \cdot u_{m}^{i} u_{m}^{i} d x \\
& +\frac{1}{\lambda_{m}} \int_{R_{0} \leq\left|x-z_{m}\right| \leq 2 \widetilde{R}} h(x)\left|u_{m}\right|^{2} d x \\
\equiv & J_{m}^{1}+J_{m}^{2}+J_{m}^{3}+J_{m}^{4} .
\end{aligned}
$$

By (3.19), (3.21) and the fact that $Q_{m}(t)$ is nondecreasing, it is evident that

$$
\left|J_{m}^{4}\right| \leqslant Q_{m}(2 \tilde{R})-Q_{m}\left(R_{0}\right)<\alpha+1 / m-(\alpha-\epsilon)=1 / m+\epsilon<\mu(\epsilon)
$$

(for $m$ large enough).

By (2.1), (2.2) and (2.3) and since $\left\|u_{m}\right\| \leqslant C$, we have that

$$
\begin{aligned}
\left|J_{m}^{3}\right| & \leqslant 2 a_{2} \int_{R_{0} \leq\left|x-z_{m}\right| \leq 2 \widetilde{R}} \sigma\left(\left|\xi_{m} u_{m}\right|\right)\left|D \xi_{m}\right|^{2}\left|u_{m}\right|^{2} d x \\
& \leqslant \frac{C}{\hat{R}^{2}} \int_{R_{0} \leq\left|x-z_{m}\right| \leq 2 \widetilde{R}} \sigma\left(\left|u_{m}\right|\right)\left|u_{m}\right|^{2} d x \\
& \leqslant \frac{C}{\hat{R}^{2}} \int_{\mathbb{R}^{n}}\left(\left|u_{m}\right|^{2}+\left|u_{m}\right|^{q+2}\right) d x \\
& \leqslant \frac{C}{\tilde{R}^{2}}<\mu(\epsilon),
\end{aligned}
$$

for $\tilde{R}(\epsilon)$ large enough. In the same way, using (2.3) and (3.8) we have that

$$
\begin{aligned}
\left|J_{m}^{2}\right| & \leqslant \frac{C}{\tilde{R}} \int_{R_{0} \leq\left|x-z_{m}\right| \leq 2 \tilde{R}}\left|a_{\alpha \beta}\left(x, \xi_{m} u_{m}\right) D_{\alpha} u_{m}^{i} D_{\beta} u_{m}^{i}\right| d x \\
& \leqslant \frac{C}{\tilde{R}} \int_{\mathbb{R}^{n}} \sigma\left(\left|u_{m}\right|\right)\left|D u_{m}\right|\left|u_{m}\right| \\
& \leqslant \frac{C}{\tilde{R}} \int_{\mathbb{R}^{n}} \sigma\left(\left|u_{m}\right|\right)\left(\left|D u_{m}\right|^{2}+\left|u_{m}\right|^{2}\right) d x \\
& <\frac{C}{\tilde{R}}<\mu(\epsilon)
\end{aligned}
$$


for $\tilde{R}(\epsilon)$ large enough. By (2.1), (3.19), (3.21) and (3.22) we have that

$$
\begin{aligned}
0 & \leqslant J_{m}^{1} \leqslant C \int_{R_{0} \leq\left|x-z_{m}\right| \leq 2 \tilde{R}} \sigma\left(\left|u_{m}\right|\right)\left|D u_{m}\right|^{2} \\
& \leqslant C \int_{R_{0} \leq\left|x-z_{m}\right| \leq 2 \tilde{R}} a_{\alpha \beta}\left(x, u_{m}\right) D_{\alpha} u_{m}^{i} D_{\beta} u_{m}^{i} \\
& \leqslant Q_{m}\left(2 R_{m}\right)-Q_{m}\left(R_{0}\right)<\alpha+1 / m-(\alpha-\epsilon) \\
& =1 / m+\epsilon<\mu(\epsilon)
\end{aligned}
$$

(for $m$ large enough).

Combining the above estimates, we see that (3.24) holds and (3.13) holds by (3.23). Similarly, (3.16) holds.

It is easy to show (see e.g. Lemma I.1 of [4]) that

(3.26) $\left|\int_{\left|x-z_{m}\right| \geq 2 R_{m}} \frac{1}{\lambda_{m}}\left[a_{\alpha \beta}\left(x, u_{m}\right) D_{\alpha} u_{m}^{i} D_{\beta} u_{m}^{i}+h(x)\left|u_{m}\right|^{2}\right] d x-(1-\alpha)\right|<\mu(\epsilon)$

On the other hand, we have

$$
\begin{aligned}
\frac{1}{\lambda_{m}} I\left(u_{m}^{2}\right)= & \frac{1}{\lambda_{m}} \int_{\left|x-z_{m}\right| \geq R_{m}}\left[a_{\alpha \beta}\left(x, u_{m}^{2}\right) D_{\alpha}\left(u_{m}^{2}\right)^{i} D_{\beta}\left(u_{m}^{2}\right)^{i}+h(x)\left|u_{m}^{2}\right|^{2}\right] d x \\
= & \frac{1}{\lambda_{m}} \int_{R_{m} \leq\left|x-z_{m}\right| \leq 2 R_{m}}\left[a_{\alpha \beta}\left(x, u_{m}^{2}\right) D_{\alpha}\left(u_{m}^{2}\right)^{i} D_{\beta}\left(u_{m}^{2}\right)^{i}+h(x)\left|u_{m}^{2}\right|^{2}\right] d x \\
& +\frac{1}{\lambda_{m}} \int_{\left|x-z_{m}\right| \geq 2 R_{m}}\left[a_{\alpha \beta}\left(x, u_{m}\right) D_{\alpha} u_{m}^{i} D_{\beta} u_{m}^{i}+h(x)\left|u_{m}\right|^{2}\right] d x
\end{aligned}
$$

Similarly to (3.24), we can prove that

$$
\text { (3.28) } \frac{1}{\lambda_{m}} \int_{R_{m} \leq\left|x-z_{m}\right| \leq 2 R_{m}}\left[a_{\alpha \beta}\left(x, u_{m}^{2}\right) D_{\alpha}\left(u_{m}^{2}\right)^{i} D_{\beta}\left(u_{m}^{2}\right)^{i}+h(x)\left|u_{m}\right|^{2}\right] d x \leqslant \mu(\epsilon)
$$

Thus (3.26) and (3.27) imply that (3.14) holds. Similarly, (3.17) holds.

By (3.19) and (3.21) we have that

$$
\begin{aligned}
\left\|u_{m}-\left(u_{m}^{1}+u_{m}^{2}\right)\right\|_{2}^{2} & =\int_{\mathbb{R}^{n}}\left|1-\xi_{m}-\varphi_{m}\right|^{2}\left|u_{m}\right|^{2} d x \\
& \leqslant C \int_{\tilde{R} \leq\left|x-z_{m}\right| \leq 2 R_{m}}\left|u_{m}\right|^{2} \\
& \leqslant C\left[Q_{m}\left(2 R_{m}\right)-Q_{m}\left(R_{0}\right)\right]<\mu(\epsilon) .
\end{aligned}
$$


So we have (3.11). Similarly, by $\left\|u_{m}\right\| \leqslant C$ and $\left\|u_{m}^{1}\right\| \leqslant C,\left\|u_{m}^{2}\right\| \leqslant C$, we see that (3.12) holds.

Finally we prove (3.15). Since

$$
\begin{aligned}
I\left(u_{m}\right) \geqslant & \int_{\left|x-z_{m}\right| \leq \tilde{R}}\left[a_{\alpha \beta}\left(x, u_{m}\right) D_{\alpha} u_{m}^{i} D_{\beta} u_{m}^{i}+h(x)\left|u_{m}\right|^{2}\right] d x \\
& +\int_{\left|x-z_{m}\right| \geq 2 R_{m}}\left[a_{\alpha \beta}\left(x, u_{m}\right) D_{\alpha} u_{m}^{i} D_{\beta} u_{m}^{i}+h(x)\left|u_{m}\right|^{2}\right] d x \\
= & I\left(u_{m}^{1}\right)+I\left(u_{m}^{2}\right) \\
& -\int_{\tilde{R} \leq\left|x-z_{m}\right| \leq 2 \tilde{R}}\left[a_{\alpha \beta}\left(x, u_{m}^{1}\right) D_{\alpha}\left(u_{m}^{1}\right)^{i} D_{\beta}\left(u_{m}^{1}\right)^{i}+h(x)\left|u_{m}^{1}\right|^{2}\right] d x \\
& -\int_{R_{m} \leq\left|x-z_{m}\right| \leq 2 R_{m}}\left[a_{\alpha \beta}\left(x, u_{m}^{2}\right) D_{\alpha}\left(u_{m}^{2}\right)^{i} D_{\beta}\left(u_{m}^{2}\right)^{i}+h(x)\left|u_{m}^{2}\right|^{2}\right] d x
\end{aligned}
$$

and because of (3.24) and (3.28), we deduce that

$$
I\left(u_{m}\right) \geqslant I\left(u_{m}^{1}\right)+I\left(u_{m}^{2}\right)-\mu(\epsilon) .
$$

Thus (3.15) holds. Similarly (3.18) holds.

Lemma 3.4. (cf. Lemma 1.1 of [5].) Let $1<p \leqslant \infty, 1 \leqslant q<\infty$, with $q \neq N p /$ $(N-p)$ if $p<N$. Assume that $\left(u_{m}\right)$ is bounded in $L^{q}\left(\mathbb{R}^{N}\right),\left|D u_{m}\right|$ is bounded in $L^{p}\left(\mathbb{R}^{N}\right)$ and

$$
\sup _{y \in \mathbb{R}^{N}} \int_{y+B_{R}}\left|u_{m}\right|^{q} d x \rightarrow 0 \text { as } m \rightarrow \infty \text {, for some } R>0 .
$$

Then $u_{m} \rightarrow 0$ in $L^{t}\left(\mathbb{R}^{N}\right)$ for any $t$ between $q$ and $N p /(N-p)$.

We now turn to prove Theorem 2.1 and Theorem 2.2. We already know that there is a minimizing sequence $\left(u_{m}\right)$ of $I_{\lambda}$ (or $\left.I_{\lambda}^{\infty}\right)$ such that Lemma 3.3 holds. If «vanishing» occurs, then

$$
\lim _{m \rightarrow \infty} \sup _{y \in \mathbb{R}^{n}} \int_{y+B_{R}}\left[a_{\alpha \beta}\left(x, u_{m}\right) D_{\alpha} u_{m}^{i} D_{\beta} u_{m}^{i}+h(x)\left|u_{m}\right|^{2}\right] d x=0
$$

for all $R$. We know also that $\left(D u_{m}\right)$ is bounded in $L^{2}\left(\mathbb{R}^{n}\right)$ and by (3.29) we know that

$$
\lim _{m \rightarrow \infty} \sup _{y \in \mathbb{R}^{n}} \int_{y+B_{R}}\left|u_{m}\right|^{2} d x=0 \quad \text { (for any } R>0 \text { ). }
$$


So Lemma 3.4 gives that

$$
\lim _{m \rightarrow+\infty} \int_{\mathbb{R} n}\left|u_{m}\right|^{p} d x=0
$$

and this contradicts

$$
\int_{\mathbb{R} n}\left|u_{m}\right|^{p} d x=\lambda
$$

Thus we have ruled out «vanishing».

If «dichotomy» occurs, then Lemma 3.3 shows that for any $\epsilon>0$, there are $u_{m}^{1}, u_{m}^{2} \in E$ such that (3.10)-(3.15) hold (or (3.10), (3.12), (3.5) and (3.18) hold in the case of $\left.I_{\lambda}^{\infty}\right)$. Therefore we would have that

$$
\begin{aligned}
I_{\lambda}+\epsilon & \geqslant I\left(u_{m}\right) \\
& \geqslant I\left(u_{m}^{1}\right)+I\left(u_{m}^{2}\right)-\mu(\epsilon) \\
& \geqslant I_{\int_{\mathbb{R}^{n}}\left|u_{m}^{1}\right| p d x}+I_{\int_{\mathbb{R}^{n}}\left|u_{m}^{2}\right|^{p} d x}-\mu(\epsilon) .
\end{aligned}
$$

We may assume that

$$
\lim _{m \rightarrow \infty} \int_{\mathbb{R}^{n}}\left|u_{m}^{1}\right|^{p} d x=\lambda_{1}(\epsilon), \quad \lim _{m \rightarrow \infty} \int_{\mathbb{R}^{n}}\left|u_{m}^{2}\right|^{p} d x=\lambda_{2}(\epsilon)
$$

Now

$$
\lambda=\int_{\mathbb{R}^{n}}\left|u_{m}\right|^{p} d x
$$

and

$$
\begin{aligned}
\left.\left|\int_{\mathbb{R}^{n}}\right| u_{m}\right|^{p} d x-\int_{\mathbb{R}^{n}}\left|u_{m}^{1}\right|^{p} d x-\int_{\mathbb{R}^{n}}\left|u_{m}^{2}\right|^{p} d x \mid & \leqslant \int_{\mathbb{R}^{n}}\left|1-\varphi_{m}^{p}-\xi_{m}^{p}\right|\left|u_{m}\right|^{p} d x \\
& \leqslant C \int_{R_{0} \leq\left|x-z_{m}\right| \leq 2 R_{m}}\left|u_{m}\right|^{p} d x \\
& \leqslant C\left(\int_{R_{0} \leq\left|x-z_{m}\right| \leq 2 R_{m}}\left|u_{m}\right|^{2} d x\right)^{p / 2} \\
& <\mu(\epsilon),
\end{aligned}
$$

(where we have made use of notations in the proof of Lemma 3.3.)

We conclude that

$$
\left|\lambda-\left(\lambda_{1}(\epsilon)+\lambda_{2}(\epsilon)\right)\right| \leqslant \mu(\epsilon)
$$

Letting $m \rightarrow \infty$ in (3.30) and using Lemma 3.1 we obtain that

$$
I_{\lambda}+\epsilon \geqslant I_{\lambda_{1}(\epsilon)}+I_{\lambda_{2}(\epsilon)}-\mu(\epsilon)
$$


We assume now that $\lambda_{1}(\epsilon) \rightarrow \lambda_{1}, \lambda_{2}(\epsilon) \rightarrow \lambda_{2}$ as $\epsilon \rightarrow 0$. Then we have by Lemma 3.1 , that

$$
I_{\lambda} \geqslant I_{\lambda_{1}}+I_{\lambda_{2}}
$$

By Lemma 3.3 and the fact that $\lambda_{m} \geqslant c>0$ we have that

$$
\begin{aligned}
& \left|I\left(u_{m}^{1}\right)-\tilde{\alpha}\right|<\mu(\epsilon), \quad \text { where } \tilde{\alpha}>0 \\
& \left|I\left(u_{m}^{2}\right)-\tilde{\beta}\right|<\mu(\epsilon), \quad \text { where } \beta>0 .
\end{aligned}
$$

Thus, if $\lambda_{1}=0$ then by (3.31) $\lambda_{2}=\lambda$. Since

$$
I_{\lambda}+\epsilon \geqslant I\left(u_{m}\right) \geqslant I\left(u_{m}^{1}\right)+I\left(u_{m}^{2}\right)-\mu(\epsilon)
$$

we obtain that

$$
I_{\lambda} \geqslant \tilde{\alpha}+I_{\lambda_{2}(\epsilon)}-\mu(\epsilon)
$$

Hence

$$
I_{\lambda} \geqslant \tilde{\alpha}+I_{\lambda}
$$

This is a contradiction and so $\lambda_{1}>0$; similarly $\lambda_{2}>0$. And now $\lambda_{1}+\lambda_{2}=\lambda$ and (3.32) contradict (3.5). Thus we have ruled out the «dichotomy» for $I_{\lambda}$. Similarly we can rule out the "dichotomy» for $I_{\lambda}^{\infty}$ using (3.4).

So we only have «compactness» i.e. there exists $\left(y_{m}\right) \subset \mathbb{R}^{n}$ such that for any $\epsilon>0$ there exists $R=R(\epsilon)>0$ with

$$
\int_{\left|x-y_{m}\right| \leq R}\left[a_{\alpha \beta}\left(x, u_{m}\right) D_{\alpha} u_{m}^{i} D_{\beta} u_{m}^{i}+h(x)\left|u_{m}\right|^{2}\right] d x \geqslant \lambda_{m}(1-\epsilon)
$$

Hence

$$
\begin{array}{r}
\int_{\left|x-y_{m}\right| \geq R}\left[a_{\alpha \beta}\left(x, u_{m}\right) D_{\alpha} u_{m}^{i} D_{\beta} u_{m}^{i}+h(x)\left|u_{m}\right|^{2}\right] d x \leqslant \lambda_{m} \epsilon \\
\int_{\left|x-y_{m}\right| \geq R}\left[\left|D u_{m}\right|^{2}+\left|u_{m}\right|^{2}\right] d x \leqslant \mu(\epsilon)
\end{array}
$$

or, in the case of $I_{\lambda}^{\infty}$, we have

$$
\begin{array}{r}
\int_{\left|x-y_{m}\right| \geq R}\left[\bar{a}_{\alpha \beta}\left(u_{m}\right) D_{\alpha} u_{m}^{i} D_{\beta} u_{m}^{i}+\bar{h}\left|u_{m}\right|^{2}\right] d x \leqslant \lambda_{m} \epsilon \\
\int_{\left|x-y_{m}\right| \geq R}\left[\left|D u_{m}\right|^{2}+\left|u_{m}\right|^{2}\right] d x \leqslant \mu(\epsilon)
\end{array}
$$

We first prove Theorem 2.1. Let $\bar{u}_{m}(x)=u_{m}\left(x+y_{m}\right)$, then $\left\|\bar{u}_{m}\right\| \leqslant C<+\infty$ and by (3.34) and the Sobolev embedding theorem we may assume the existence of a $u=\left(u^{1}, u^{2}, \ldots, u^{N}\right) \in E$ such that 


$$
\left\{\begin{array}{ll}
\bar{u}_{m} \rightarrow u & \text { in } E \\
\bar{u}_{m}^{i} \rightarrow u^{i} & \text { in } H^{1}\left(R^{n}\right) \\
\bar{u}_{m}^{i} \rightarrow u^{i} & \text { in } L^{t}\left(R^{n}\right) \\
\bar{u}_{m} \rightarrow u & \text { a.e. in } R^{n},
\end{array} \quad 2 \leqslant t<2 \hat{n} /(\hat{n}-2)\right.
$$

for $1 \leqslant i \leqslant N$, and

$$
\lambda=\int_{\mathbb{R}^{n}}\left|u_{m}\right|^{p} d x=\int_{\mathbb{R}^{n}}\left|\bar{u}_{m}\right|^{p} d x \rightarrow \int_{\mathbb{R} n}|u|^{p} d x \quad(\text { as } \quad m \rightarrow \infty) .
$$

Also

$$
I_{\lambda}^{\infty}=\lim _{m \rightarrow \infty} \int_{\mathbb{R}^{n}}\left[\bar{a}_{\alpha \beta}\left(\bar{u}_{m}\right) D_{\alpha} \bar{u}_{m}^{i} D_{\beta} \bar{u}_{m}^{i}+\bar{h}\left|u_{m}\right|^{2}\right] d x .
$$

By (3.35) and (ii), (iii) of Section 2 we see that

$$
\bar{a}_{\alpha \beta}\left(\bar{u}_{m}\right) \rightarrow \bar{a}_{\alpha \beta}(u) \text { a.e. in } \mathbb{R}^{n} .
$$

So for any bounded domain $\Omega \subset \mathbb{R}^{n}$ and $\delta>0$, there is a $\Omega_{\delta} \subset \Omega$ with

$$
\left|\Omega-\Omega_{\delta}\right|<\delta
$$

and

$$
\bar{a}_{\alpha \beta}\left(\bar{u}_{m}\right) \rightarrow \bar{a}_{\alpha \beta}(u)
$$

uniformly for $x \in \Omega_{\delta}$ where $|A|$ denotes the Lebesgue measure of $A$ for any $A \subset \mathbb{R}^{n}$. So that for any $\epsilon>0$ and $m$ large enough we have, by (2.2), that

$$
\begin{aligned}
\int_{\Omega} \bar{a}_{\alpha \beta}\left(\bar{u}_{m}\right) D_{\alpha} \bar{u}_{m}^{i} D_{\beta} \bar{u}_{m}^{i} d x \geqslant & \int_{\Omega_{\delta}} \bar{a}_{\alpha \beta}\left(\bar{u}_{m}\right) D_{\alpha} \bar{u}_{m}^{i} D_{\beta} \bar{u}_{m}^{i} d x \\
\geqslant & \int_{\Omega_{\delta}}\left[\bar{a}_{\alpha \beta}\left(\bar{u}_{m}^{i}\right)-\bar{a}_{\alpha \beta}(u)\right] D_{\alpha} \bar{u}_{m}^{i} D_{\beta} \bar{u}_{m}^{i} d x \\
& +\int_{\Omega_{\delta}} a_{\alpha \beta}(u) D_{\alpha} \bar{u}_{m}^{i} D_{\beta} \bar{u}_{m}^{i} d x \\
\geqslant & -\epsilon \int_{\mathbb{R} n}\left|D \bar{u}_{m}\right|^{2} d x+\int_{\Omega_{\delta}} a_{\alpha \beta}(u) D_{\alpha} \bar{u}_{m}^{i} D_{\beta} \bar{u}_{m}^{i} d x \\
\geqslant & -\epsilon C+\int_{\Omega_{\delta}} a_{\alpha \beta}(u) D_{\alpha} \bar{u}_{m}^{i} D_{\beta} \bar{u}_{m}^{i} d x .
\end{aligned}
$$

By (3.35), Mazur's theorem (see [6]) and Fatou's lemma, we see that

$$
\liminf _{m \rightarrow \infty} \int_{\Omega_{\delta}} \bar{a}_{\alpha \beta}(u) D_{\alpha} \bar{u}_{m}^{i} D_{\beta} \bar{u}_{m}^{i} d x \geqslant \int_{\Omega_{\delta}} \bar{a}_{\alpha \beta}(u) D_{\alpha} u^{i} D_{\beta} u^{i} d x
$$


and hence we get, for any $N$, that

$$
\begin{aligned}
\liminf _{m \rightarrow \infty} \int_{\Omega} \bar{a}_{\alpha \beta}\left(\bar{u}_{m}\right) D_{\alpha} \bar{u}_{m}^{i} D_{\beta} \bar{u}_{m}^{i} d x & \geqslant \int_{\Omega_{\delta}} \bar{a}_{\alpha \beta}(u) D_{\alpha} u^{i} D_{\beta} u^{i} d x \\
& \geqslant \int_{\Omega_{\delta}}\left[\bar{a}_{\alpha \beta}(u) D_{\alpha} u^{i} D_{\beta} u^{i}\right]_{N} d x
\end{aligned}
$$

where the function $[f]_{N}$ for any $f \geqslant 0$ is given by

$$
[f]_{N}=\left\{\begin{array}{lll}
f & \text { if } & f \leqslant N \\
N & \text { if } & f \geqslant N
\end{array}\right.
$$

Since $\left[\bar{a}_{\alpha \beta}(u) D_{\alpha} u^{i} D_{\beta} u^{i}\right]_{N} \in L^{1}(\Omega)$, and since $\left|\Omega_{\delta}\right| \rightarrow|\Omega|$ we have that

$$
\liminf _{m \rightarrow \infty} \int_{\Omega} \bar{a}_{\alpha \beta}\left(\bar{u}_{m}\right) D_{\alpha} \bar{u}_{m}^{i} D_{\beta} \bar{u}_{m}^{i} d x \geqslant \int_{\Omega}\left[\bar{a}_{\alpha \beta}(u) D_{\alpha} u^{i} D_{\beta} u^{i}\right]_{N} d x .
$$

Letting $N \rightarrow \infty$, we have that

$$
\liminf _{m \rightarrow \infty} \int_{\Omega} \bar{a}_{\alpha \beta}\left(\bar{u}_{m}\right) D_{\alpha} u_{m}^{i} D_{\beta} u_{m}^{i} \geqslant \int_{\Omega} \bar{a}_{\alpha \beta}(u) D_{\alpha} u^{i} D_{\beta} u^{i} d x
$$

Thus, since the supremum of any sequence of lower-semicontinuous functions is still lower-semicontinuous, we have that

$$
\liminf _{m \rightarrow \infty} \int_{\mathbb{R}^{n}} \bar{a}_{\alpha \beta}\left(\bar{u}_{m}\right) D_{\alpha} u_{m}^{i} D_{\beta} u_{m}^{i} d x \geqslant \int_{\mathbb{R} n} \bar{a}_{\alpha \beta}(u) D_{\alpha} u^{i} D_{\beta} u^{i} d x
$$

On the other hand, by (3.35) we have that

$$
\lim _{m \rightarrow \infty} \int_{\mathbb{R}^{n}} \bar{h}\left|u_{m}\right|^{2} d x=\int_{\mathbb{R}^{n}} \bar{h}|u|^{2} d x
$$

and so we get that

$$
\begin{aligned}
I_{\lambda}^{\infty} & \geqslant \liminf _{m \rightarrow \infty} \int_{\mathbb{R}^{n}} \bar{a}_{\alpha \beta}\left(\bar{u}_{m}\right) D_{\alpha} u_{m}^{i} D_{\beta} u_{m}^{i} d x+\lim _{m \rightarrow \infty} \int_{\mathbb{R} n} \bar{h}\left|u_{m}\right|^{2} d x \\
& \geqslant \int_{\mathbb{R} n}\left[\bar{a}_{\alpha \beta}(u) D_{\alpha} u^{i} D_{\beta} u^{i}+\bar{h}|u|^{2}\right] d x
\end{aligned}
$$

But

$$
\int_{\mathbb{R}^{n}}|u|^{p} d x=\lambda
$$


and so

$$
I_{\lambda}^{\infty}=\int_{\mathbb{R}^{n}}\left[\bar{a}_{\alpha \beta}(u) D_{\alpha} u^{i} D_{\beta} u^{i}+\bar{h}|u|^{2}\right] d x
$$

so $I_{\lambda}^{\infty}$ is achieved and Theorem 2.1 is proved.

In the case of $I_{\lambda}$, by (3.33) we still have (3.35) with $\bar{u}_{m}(x)=u_{m}\left(x+y_{m}\right)$. If there is $\lambda_{0} \in(0, \lambda]$ such that $I_{\lambda_{0}}=I_{\lambda_{0}}^{\infty}$, then by Theorem 2.1 there exists $u_{0} \in E$ with $\int_{\mathbb{R}^{n}}\left|u_{0}\right|^{p} d x=\lambda_{0}$ and such that $I_{\lambda_{0}}^{\infty}=I^{\infty}\left(u_{0}\right)$, and hence $I_{\lambda_{0}} \leqslant I\left(u_{0}\right) \leqslant$ $I^{\infty}\left(u_{0}\right)=I_{\lambda_{0}}^{\infty}=I_{\lambda_{0}}$ implies that $I\left(u_{0}\right)=I_{\lambda_{0}}$ and therefore $I_{\lambda_{0}}$ is achieved by $u_{0}$ Theorem 2.2 is proved.

Now we assume that for any $0<\mu \leqslant \lambda$ we have $I_{\mu}<I_{\mu}^{\infty}$. If $\left(y_{m}\right)$ is unbounded, say $\left|y_{m}\right| \rightarrow \infty$, we have, by (ii) of Section 2 and (3.35), that

$$
a_{\alpha \beta}\left(x+y_{m}, \bar{u}_{m}\right) \rightarrow \bar{a}_{\alpha \beta}(u) \text { a.e. in } \mathbb{R}^{n} \text {. }
$$

So we have, as in (3.37), that

$$
\liminf _{i \rightarrow \infty} \int_{\mathbb{R}^{n}} a_{\alpha \beta}\left(x+y_{m}, \bar{u}_{m}\right) D_{\alpha} u_{m}^{i} D_{\beta} u_{m}^{i} d x \geqslant \int_{\mathbb{R}^{n}} \bar{a}_{\alpha \beta}(u) D_{\alpha} u^{i} D_{\beta} u^{i} d x
$$

By (v) of Section 2, (3.35) and the Lebesgue's theorem we have that

$$
\lim _{m \rightarrow \infty} \int_{\mathbb{R}^{n}} h\left(x+y_{m}\right)\left|\bar{u}_{m}\right|^{2} d x=\int_{\mathbb{R} n} \bar{h}|u|^{2} d x
$$

Combining (3.38), (3.39) and

$$
\int_{\mathbb{R}^{n}}|u|^{p} d x=\lambda
$$

we have that

$$
\begin{aligned}
I_{\lambda} & =\lim _{m \rightarrow \infty} \int_{\mathbb{R}^{n}}\left[a_{\alpha \beta}\left(x, u_{m}\right) D_{\alpha} u_{m}^{i} D_{\beta} u_{m}^{i}+h(x)\left|u_{m}\right|^{2}\right] d x \\
& =\lim _{m \rightarrow \infty} \int_{\mathbb{R}^{n}}\left[a_{\alpha \beta}\left(x+y_{m}, \bar{u}_{m}\right) D_{\alpha} \bar{u}_{m}^{i} D_{\beta} \bar{u}_{m}^{i}+h\left(x+y_{m}\right)\left|\bar{u}_{m}\right|^{2}\right] d x \\
& \geqslant \int_{\mathbb{R}^{n}}\left[\bar{a}_{\alpha \beta}(u) D_{\alpha} u^{i} D_{\beta} u^{i}+\bar{h}|u|^{2}\right] d x \\
& \geqslant I_{\lambda}^{\infty}
\end{aligned}
$$

which contradicts that $I_{\mu}<I_{\mu}^{\infty}$ for any $0<\mu \leqslant \lambda$. Thus we have $\left|y_{m}\right| \leqslant C$ and by (3.34) we see that for any $\epsilon>0$, there is a $R(\epsilon)>0$ such that

$$
\int_{|x| \geq R}\left[\left|D u_{m}\right|^{2}+\left|u_{m}\right|^{2}\right] d x \leqslant \epsilon
$$


and hence we may assume the existence of a $u_{0} \in E$ such that

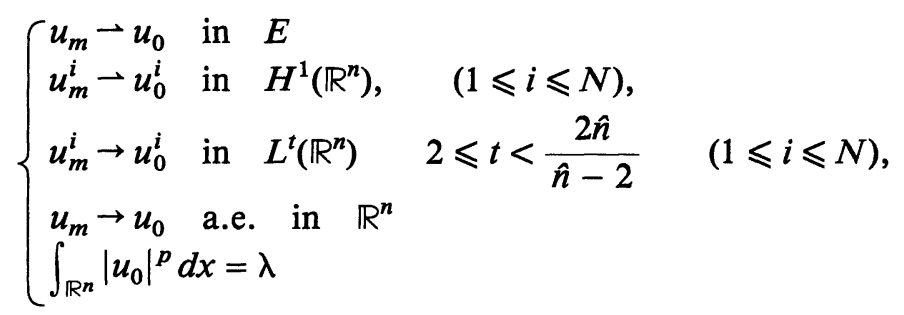

Thus, similarly to (3.38) and (3.39) we can prove that

$$
\begin{aligned}
\liminf _{m \rightarrow \infty} \int_{\mathbb{R}^{n}} a_{\alpha \beta}\left(x, u_{m}\right) D_{\alpha} u_{m}^{i} D_{\beta} u_{m}^{i} d x & \geqslant \int_{\mathbb{R}^{n}} a_{\alpha \beta}\left(x, u_{0}\right) D_{\alpha} u_{0}^{i} D_{\beta} u_{0}^{i} d x \\
\lim _{m \rightarrow \infty} \int_{\mathbb{R}^{n}} h(x)\left|u_{m}\right|^{2} d x & =\int_{\mathbb{R}^{n}} h(x)\left|u_{0}\right|^{2} d x .
\end{aligned}
$$

Since $\int_{\mathbb{R}^{n}}\left|u_{0}\right|^{p} d x=\lambda$ we have

$$
\begin{aligned}
I_{\lambda} & \geqslant \liminf _{m \rightarrow \infty} \int_{\mathbb{R}^{n}}\left[a_{\alpha \beta}\left(x, u_{m}\right) D_{\alpha} u_{m}^{i} D_{\beta} u_{m}^{i}+h(x)\left|u_{m}\right|^{2}\right] d x \\
& \geqslant \int_{\mathbb{R}^{n}}\left[a_{\alpha \beta}\left(x, u_{0}\right) D_{\alpha} u_{0}^{i} D_{\beta} u_{0}^{i}+h(x)\left|u_{0}\right|^{2}\right] d x \\
& \geqslant I_{\lambda}
\end{aligned}
$$

and hence $I_{\lambda}$ is achieved by $u_{0} \in E$. Theorem 2.2 is proved.

\section{Proof of Theorem 2.3}

In this section, we prove Theorem 2.3. The main difficulty is that $I_{\lambda}$ is in general not in $C^{1}(E, \mathbb{R})$. To overcome this difficulty, we first prove that

$$
\left.\frac{d}{d t} I\left(\frac{\lambda(u+t \varphi)}{\|u+t \varphi\|_{p}}\right)\right|_{t=0}
$$

exists for special $\varphi \in E$ and then show that $\|u\|_{\infty}$ is finite where $u$ is a minimizer of $I_{\lambda}$ for some $\lambda>0$. Finally we prove the theorem.

Proof of TheOREM 2.3. By Theorem 2.2 we may assume without loss of generality the existence of $u \in E$, with $\int_{\mathbb{R}^{n}}|u|^{p} d x=1$ and such that

$$
I_{1}=\int_{\mathbb{R}^{n}}\left[a_{\alpha \beta}(x, u) D_{\alpha} u^{i} D_{\beta} u^{i}+h(x)|u|^{2}\right] d x .
$$


We first prove that for any $\tau \geqslant 0$,

$$
\left.\frac{d}{d t} I\left(\frac{u+t|u|_{L}^{\tau} u}{\left\|u+t|u|_{L}^{\tau} u\right\|_{p}}\right)\right|_{t=0}=0
$$

where

$$
|u|_{L}=\left\{\begin{array}{ccc}
|u| & \text { if } & |u| \leqslant L \\
L & \text { if } & |u| \geqslant L
\end{array}\right.
$$

It is easy to see that $u+t|u|_{L}^{\tau} u=\left(1+t|u|_{L}^{\tau}\right) u \in E$ for any $t \geqslant 0$ and since $u$ achieves $I_{1},(4.1)$ will hold if

$$
\left.\frac{d}{d t} I\left(\frac{u+t|u|_{L}^{\tau} u}{\left\|u+t|u|_{L}^{\tau} u\right\|_{p}}\right)\right|_{t=0}
$$

exists.

Because $0 \leqslant|u|_{L}^{\tau} \leqslant L^{\tau}$, there is a $M>0$, depending on $\beta$ and $L$, such that

$$
\frac{1}{2} \leqslant\left\|u+t|u|_{L}^{\tau} u\right\|_{p} \leqslant M
$$

for $t$ small enough.

It is easy to prove that

$$
\left.\frac{d}{d t}\left(\left\|u+t|u|_{L}^{\tau} u\right\|_{p}\right)\right|_{t=0}=\int_{\mathbb{R}^{n}}|u|^{p}|u|_{L}^{\tau} d x
$$

and hence

$$
\text { (4.4) } \begin{aligned}
\left.\frac{d}{d t}\left[\int_{\mathbb{R}^{n}} \frac{\left.\left.h(x)|u+t| u\right|_{L} ^{\tau} u\right|^{2}}{\left\|u+t|u|_{L}^{\tau} u\right\|_{p}} d x\right]\right|_{t=0} \\
\quad=2 \int_{\mathbb{R}^{n}} h(x)|u|^{2}|u|_{L}^{\tau} d x-2 \int_{\mathbb{R}^{n}} h(x)|u|^{2} d x \int_{\mathbb{R}^{n}}|u|^{p}|u|_{L}^{\tau} d x .
\end{aligned}
$$

On the other hand

$$
\begin{aligned}
I\left(\frac{u+t|u|_{L}^{\tau} u}{\left\|u+t|u|_{L}^{\tau} u\right\|_{p}}\right)= & \int_{\mathbb{R}^{n}} a_{\alpha \beta}\left(x, \frac{u+t|u|_{L}^{\tau} u}{\left\|u+t|u|_{L}^{\tau} u\right\|_{p}}\right) \frac{D_{\alpha} u^{i} D_{\beta} u^{i}}{\left\|u+t|u|_{L}^{\tau} u\right\|_{p}^{2}} d x \\
& +2 t \int_{\mathbb{R}^{n}} a_{\alpha \beta}\left(x, \frac{u+t|u|_{L}^{2} u}{\left\|u+t|u|_{L}^{\tau} u\right\|_{p}}\right) \frac{D_{\alpha} u^{i} D_{\beta}|u|_{L}^{\tau} u^{i}}{\left\|u+t|u|_{L}^{\tau} u\right\|_{p}^{2}} d x \\
& +t^{2} \int_{\mathbb{R}^{n}} a_{\alpha \beta}\left(\frac{u+t|u|_{L}^{\tau} u}{\left\|u+t|u|_{L}^{\tau} u\right\|_{p}}\right) \frac{D_{\alpha}\left(|u|_{L}^{\tau} u^{i}\right) D_{\beta}\left(|u|_{L}^{\tau} u^{i}\right)}{\left\|u+t|u|_{L}^{\tau} u\right\|_{p}^{2}} d x \\
& +\int_{\mathbb{R}^{n}} \frac{\left.\left.h(x)|u+t| u\right|_{L} ^{\tau} u\right|^{2}}{\left\|u+t|u|_{L}^{\tau} u\right\|_{p}^{2}} d x
\end{aligned}
$$


(4.5) $I\left(\frac{u+t|u|_{L}^{\tau} u}{\left\|u+t|u|_{L}^{\tau} u\right\|_{p}}\right)=I^{1}(t)+I^{2}(t)+I^{3}(t)+\int_{\mathbb{R}^{n}} \frac{\left.\left.h(x)|u+t| u\right|_{L} ^{\tau} u\right|^{2}}{\left\|u+t|u|_{L}^{\tau} u\right\|_{p}^{2}} d x$

Using (ii), (iii) of Section 2, (4.2) and (2.1), the inequality $\left|a_{\alpha \beta}\left(x, \frac{u+t|u|_{L}^{\tau} u}{\left\|u+t|u|_{L}^{\tau}\right\|_{p}}\right) \frac{u^{i} t|u|^{\tau-1} D_{\alpha} u^{2} D_{\beta}|u|_{L}}{\left\|u+t|u|_{L}^{\tau} u\right\|_{p}^{2}}\right| \leqslant\left. C\left|D_{\alpha} u^{i} D_{\beta}\right| u\right|_{L}|u|^{\tau} \mid, L^{1}\left(\mathbb{R}^{n}\right)$ (which holds if $|u| \leqslant L$ ) and the Dominated Convergence Theorem, we have that

$$
\left.\frac{d}{d t}\left[I^{2}(t)\right]\right|_{t=0}=\lim _{t \rightarrow 0} \frac{I^{2}(t)}{t}=2 \int_{\mathbb{R}^{n}} a_{\alpha \beta}(x, u) D_{\alpha} u^{i} D_{\beta}\left(|u|_{L}^{\tau} u^{i}\right) d x .
$$

Similarly, we have that

$$
\left.\frac{d}{d t}\left[I^{3}(t)\right]\right|_{t=0}=0
$$

On the other hand

$$
\begin{aligned}
\left.\frac{d}{d t}\left[I^{1}(t)\right]\right|_{t=0}=\lim _{t \rightarrow 0} \int_{\mathbb{R}^{n}} \frac{1}{t}\left[a_{\alpha \beta}\left(x, \frac{u+t|u|_{L}^{\tau} u}{\left\|u+t|u|_{L}^{\tau}\right\|_{p}}\right)\left\|u+t|u|_{L}^{\tau} u\right\|_{p}^{-2}\right. \\
\left.\quad-a_{\alpha \beta}(x, u)\right] D_{\alpha} u^{i} D_{\beta} u^{i} d x \\
=\lim _{t \rightarrow 0} \int_{\mathbb{R}^{n}} \frac{1}{t}\left[a_{\alpha \beta}\left(x, \frac{u+t|u|_{L}^{\tau} u}{\left\|u+t|u|_{L}^{\tau} u\right\|_{p}}\right)-a_{\alpha \beta}(x, u)\right] \\
\qquad u+t|u|_{L}^{\tau} u \|_{p}^{-2} D_{\alpha} u^{i} D_{\beta} u^{i} d x \\
+\lim _{t \rightarrow 0} \frac{1}{t}\left(\left\|u+t|u|_{L}^{\tau} u\right\|_{p}^{-2}-1\right) \int_{\mathbb{R}^{n}} a_{\alpha \beta}(x, u) D_{\alpha} u^{i} D_{\beta} u^{i} d x \\
\equiv \lim _{t \rightarrow 0} I^{4}(t)+\lim _{t \rightarrow 0} I^{5}(t) .
\end{aligned}
$$

By (4.3), we have

$$
\lim _{t \rightarrow 0} I^{S}(t)=-2 \int_{\mathbb{R}^{n}}|u|^{p}|u|_{L}^{\tau} d x \int_{\mathbb{R}^{n}} a_{\alpha \beta}(x, u) D_{\alpha} u^{i} D_{\beta} u^{i} d x .
$$

Using the mean value theorem we get that

$$
\begin{aligned}
\lim _{t \rightarrow 0} I^{4}(t)=\lim _{t \rightarrow 0} \int_{\mathbb{R}^{n}} D_{u^{j}} a_{\alpha \beta}\left(x, \frac{u+t^{\prime}|u|_{L}^{\tau} u}{\left\|u+t^{\prime}|u|_{L}^{\tau} u\right\|_{p}}\right) \\
\\
{\left[\frac{|u|_{L}^{\tau} u^{j}}{\left\|u+t^{\prime}|u|_{L}^{\tau} u\right\|_{p}}-\frac{u^{j}+t^{\prime}|u|_{L}^{\tau} u^{j}}{\left\|u+t^{\prime}|u|_{L}^{\tau} u\right\|_{p}^{2}} \frac{d}{d t}\left\|u+t|u|_{L}^{\tau} u\right\|_{p \mid t=t^{\prime}}\right] } \\
\left\|u+t|u|_{L}^{\tau} u\right\|_{p}^{-2} D_{\alpha} u^{i} D_{\beta} u^{i} d x
\end{aligned}
$$




$$
\begin{aligned}
& =\lim _{t \rightarrow 0} \int_{\mathbb{R}^{n}} D_{u^{j}} a_{\alpha \beta}\left(x, \frac{u+t^{\prime}|u|_{L}^{\tau} u}{\left\|u+t^{\prime}|u|_{L}^{\tau} u\right\|_{p}}\right) \frac{|u|_{L}^{\tau} u^{j}}{\left\|u+t^{\prime}|u|_{L}^{\tau} u\right\|_{p}} \\
& \left\|u+t|u|_{L}^{\tau} u\right\|_{p}^{-2} D_{\alpha} u^{i} D_{\beta} u^{i} d x \\
& -\lim _{t \rightarrow 0} \int_{\mathbb{R}^{n}} D_{u^{j}} a_{\alpha \beta}\left(x, \frac{u+t^{\prime}|u|_{L}^{\tau} u}{\left\|u+t^{\prime}|u|_{L}^{\tau} u\right\|_{p}}\right) \\
& \frac{u^{j}+t^{\prime}|u|_{L}^{\tau} u^{j}}{\left\|u+t^{\prime}|u|_{L}^{\tau} u\right\|_{p}^{2}} \frac{d}{d t}\left\|u+t|u|_{L}^{\tau} u\right\|_{p \mid t=t^{\prime}}\left\|u+t|u|_{L}^{\tau} u\right\|_{p}^{-2} D_{\alpha} u^{i} D_{\beta} u^{i} d x \\
& \text { (4.9) } \quad \equiv \lim _{t \rightarrow 0} I^{6}(t)-\lim _{t \rightarrow 0} I^{7}(t) \quad\left(0<t^{\prime}=t^{\prime}(x)<t\right)
\end{aligned}
$$

By (vi) of Section 2 and (3.2) we have that

$$
\begin{gathered}
\left|D_{u^{j}} a_{\alpha \beta}\left(x, \frac{u+t^{\prime}|u|_{L}^{\tau} u}{\left\|u+t^{\prime}|u|_{L}^{\tau} u\right\|_{p}}\right) \frac{|u|_{L}^{\tau} u^{j}}{\left\|u+t^{\prime}|u|_{L}^{\tau} u\right\|_{p}} D_{\alpha} u^{i} D_{\beta} u^{i}\left\|u+t|u|_{L}^{\tau} u\right\|_{p}^{-2}\right| \\
\leqslant C \sigma\left(\frac{u+t^{\prime}|u|_{L}^{\tau} u}{\left\|u+t^{\prime}|u|_{L}^{\tau} u\right\|_{p}}\right) \frac{|u|_{L}^{\tau}}{1+t^{\prime}|u|_{L}^{\tau}}|D u|^{2} \\
\leqslant C \sigma(|u|)|D u|^{2} \in L^{1}\left(\mathbb{R}^{n}\right),
\end{gathered}
$$

hence by the Dominated Convergence Theorem

$$
\lim _{t \rightarrow 0} I^{6}(t)=\int_{\mathbb{R}^{n}}|u|_{L}^{\tau} u^{j} D_{u^{j}} a_{\alpha \beta}(x, u) D_{\alpha} u^{i} D_{\beta} u^{i} d x
$$

Similarly, by (vi) of Section 2, (4.2) and (4.3) we get

$$
\lim _{t \rightarrow 0} I^{7}(t)=\int_{\mathbb{R} n}|u|^{p}|u|_{L}^{\tau} d x \int_{\mathbb{R} n} u^{j} D_{u^{j}} a_{\alpha \beta}(x, u) D_{\alpha} u^{i} D_{\beta} u^{j} d x .
$$

Combining (4.4)-(4.11) we see that (4.1) holds and that

$$
\begin{aligned}
0= & \left.\frac{d}{d t} I\left(\frac{u+t|u|_{L}^{\tau} u}{\left\|u+t|u|_{L}^{\tau} u\right\|_{p}}\right)\right|_{t=0} \\
= & 2 \int_{\mathbb{R}^{n}} a_{\alpha \beta}(x, u) D_{\alpha} u^{i} D_{\beta}\left(|u|_{L}^{\tau} u^{j}\right) d x \\
& +\int_{\mathbb{R}^{n}}|u|_{L}^{\tau} u^{j} D_{u^{j}} a_{\alpha \beta}(x, u) D_{\alpha} u^{i} D_{\beta} u^{i} d x \\
& -\int_{\mathbb{R}^{n}}|u|^{p}|u|_{L}^{\tau} d x \int_{\mathbb{R}^{n}} u^{j} D_{u^{j}} a_{\alpha \beta}(x, u) D_{\alpha} u^{i} D_{\beta} u^{i} d x \\
& -2 \int_{\mathbb{R}^{n}}|u|^{p}|u|_{L}^{\tau} d x \int_{\mathbb{R}^{n}} a_{\alpha \beta}(x, u) D_{\alpha} u^{i} D_{\beta} u^{i} d x+2 \int_{\mathbb{R}^{n}} h(x)|u|^{2}|u|_{L}^{\tau} d x \\
& -2 \int_{\mathbb{R}^{n}} h(x)|u|^{2} d x \int_{\mathbb{R}^{n}}|u|^{p}|u|_{L}^{\tau} d x
\end{aligned}
$$


which implies that

$$
\begin{aligned}
& \int_{\mathbb{R}^{n}} a_{\alpha \beta}(x, u) D_{\alpha} u^{i} D_{\beta}\left(|u|_{L}^{\tau} u^{i}\right) d x+\frac{1}{2} \int_{\mathbb{R}^{n}}|u|_{L}^{\tau} u^{j} D_{u j} a_{\alpha \beta}(x, u) D_{\alpha} u^{i} D_{\beta} u^{i} d x \\
& +\int_{\mathbb{R}^{n}} h(x)|u|^{2}|u|_{L}^{\tau} d x=\lambda \int_{\mathbb{R} n}|u|^{p}|u|_{L}^{\tau} d x \quad \text { (for every } \tau \geqslant 0 \text { and } L \geqslant 0 \text { ), }
\end{aligned}
$$

where

$$
\lambda=\int_{\mathbb{R}^{n}}\left[a_{\alpha \beta}(x, u) D_{\alpha} u^{i} D_{\beta} u^{i}+\frac{1}{2} u^{j} D_{u^{j}} a_{\alpha \beta}(x, u) D_{\alpha} u^{i} D_{\beta} u^{i}+h(x)|u|^{2}\right] d x .
$$

Now we are ready to prove that $\|u\|_{\infty}<+\infty$. By (4.12), we have for any $\tau \geqslant 0$, that

$$
\begin{aligned}
& \text { (4.13) } \int_{\mathbb{R} n}|u|_{L}^{\tau} a_{\alpha \beta}(x, u) D_{\alpha} u^{i} D_{\beta} u^{j} d x+\tau \int_{\mathbb{R}^{n}}|u|_{L}^{\tau-1} u^{i} a_{\alpha \beta}(x, u) D_{\alpha} u^{i} D_{\beta}|u|_{L} d x \\
& +\frac{1}{2} \int_{\mathbb{R}^{n}}|u|_{L}^{\tau} u^{j} D_{u^{j}} a_{\alpha \beta}(x, u) D_{\alpha} u^{i} D_{\beta} u^{i} d x+\int_{\mathbb{R}^{n}} h(x)|u|^{2}|u|_{L}^{\tau} d x \\
& =\lambda \int_{\mathbb{R}^{n}}|u|^{p}|u|_{L}^{\tau} d x \text {. }
\end{aligned}
$$

It is easy to see that

$$
\begin{aligned}
\int_{\mathbb{R} n}|u|_{L}^{\tau-1} u^{i} a_{\alpha \beta}(x, u) D_{\alpha} u^{i} D_{\beta}|u|_{L} d x & =\int_{\mathbb{R} n}|u|_{L}^{\tau} a_{\alpha \beta}(x, u) D_{\alpha}|u| D_{\beta}|u|_{L} d x \\
& =\int_{\{|u| \leq L\}}|u|_{L}^{\tau} a_{\alpha \beta}(x, u) D_{\alpha}|u| D_{\beta}|u| d x \\
& \geqslant 0 .
\end{aligned}
$$

So by (2.8) we have

$$
\left(1-a_{3}\right) \int_{\mathbb{R}^{n}}|u|_{L}^{\tau} a_{\alpha \beta}(x, u) D_{\alpha} u^{i} D_{\beta} u^{i} d x \leqslant \lambda \int_{\mathbb{R}^{n}}|u|^{p}|u|_{L}^{\tau} d x
$$

hence

$$
\mu\left(1-a_{3}\right) \int_{\mathbb{R}^{n}}|D u|^{2}|u|_{L}^{\tau} d x \leqslant \lambda \int_{\mathbb{R}^{n}}|u|^{p}|u|_{L}^{\tau} d x .
$$

It is easy to see that

$$
|D| u||^{2} \leqslant|D u|^{2}
$$

and from this and (4.14) we get that

$$
\mu\left(1-a_{3}\right) \int_{\mathbb{R}^{n}}|D| u||^{2}|u|_{L}^{\tau} d x \leqslant \lambda \int_{\mathbb{R}^{n}}|u|^{p}|u|_{L}^{\tau} d x .
$$

Thus, there is a $C>0$ such that for any $\tau \geqslant 0$

$$
\left.\left.\int_{\mathbb{R}^{n}}|D| u|| u\right|_{L} ^{\tau / 2}\right|^{2} d x \leqslant C \int_{\mathbb{R}^{n}}|u|^{p}|u|_{L}^{\tau} d x
$$

holds. 
By (4.15) we have, for any $\tau \geqslant 1$, that

$$
\left.\left.\int_{\mathbb{R}^{n}}|D| u|| u\right|_{L} ^{\tau-1}\right|^{2} d x \leqslant C \int_{\mathbb{R}^{n}}|u|^{p}|u|_{L}^{2(\tau-1)} d x
$$

Let $w_{L}=|u||u|_{L}^{\tau-1}$, then we have

$$
D w_{L}=D|u||u|_{L}^{\tau-1}+(\tau-1)|u|_{L}^{\tau-2} D|u|_{L}^{\tau-2} D|u|_{L}|u| .
$$

Thus

$$
\begin{aligned}
\int_{\mathbb{R}^{n}}\left|D w_{L}\right|^{2} d x & \leqslant C\left[\left.\left.\int_{\mathbb{R}^{n}}|D| u|| u\right|_{L} ^{\tau-1}\right|^{2} d x+\left.\left.(\tau-1)^{2} \int_{\mathbb{R}^{n}}|| u\right|_{L} ^{\tau-2}|u| D|u|_{L}\right|^{2} d x\right] \\
& \leqslant C\left[\left.\left.\int_{\mathbb{R}^{n}}|D| u|| u\right|_{L} ^{\tau-1}\right|^{2} d x+\left.\left.(\tau-1)^{2} \int_{\{|u| \leq L\}}|D| u|| u\right|^{\tau-1}\right|^{2} d x\right] \\
& \leqslant C\left(1+\left.\left.(\tau-1)^{2)} \int_{\mathbb{R}^{n}}|D| u|| u\right|_{L} ^{\tau-1}\right|^{2} d x\right. \\
& \leqslant C \tau^{2} \int_{\mathbb{R}^{n}}|u|^{p}|u|_{L}^{2 \tau-2} d x .
\end{aligned}
$$

So we get

$$
\int_{\mathbb{R}^{n}}\left|D w_{L}\right|^{2} d x \leqslant C \tau^{2} \int_{\mathbb{R}^{n}}|u|^{p-2}\left|w_{L}\right|^{2} d x
$$

By (4.17), the Sobolev embedding theorems and Hölder's inequality we have that

(4.18) $\left\|w_{L}\right\|_{2^{*}}^{2} \leqslant C\left\|\left|D w_{L}\right|\right\|_{2}^{2}$

$$
\begin{aligned}
& \leqslant C \tau^{2}\left(\int_{\mathbb{R}^{n}}|u|^{(p-2) \frac{2^{*}}{p-2}} d x\right)^{\frac{p-2}{2 *}}\left(\int_{\mathbb{R}^{n}}\left|w_{L}\right|^{2} \frac{2^{* *}}{2^{*}-(p-2)} d x\right)^{\frac{2 *-(p-2)}{2}} \\
& =C \tau^{2}\|u\|_{2^{*}}^{p-2}\left\|w_{L}\right\|_{\frac{2 q}{2-2}}^{2}
\end{aligned}
$$

where $2 q /(q-2)=2 \cdot 2^{*} /\left(2^{*}-(p-2)\right)$, i.e. $q=2 \cdot 2^{*} /(p-2)$. It is easy to see that $q>n$ when $n>2$ or $n \leqslant 2$ by choosing $2^{*}$ large enough, hence $2^{*}>2^{*}>2 q /(q-2)$. If $|u|^{2} \in L^{2 q /(q-2)}\left(\mathbb{R}^{n}\right)$, letting $L \rightarrow+\infty$ in (4.18) and using the Dominated Convergence Theorem and Fatou's lemma together with the fact that $\left|w_{L}\right| \leqslant|u|^{\tau}$ we get that

$$
\left\||u|^{\tau}\right\|_{2^{*}}^{2} \leqslant C \tau^{2}\left\||u|^{\tau}\right\|_{\frac{2 q}{q-2}}^{2} .
$$

Thus $u \in L^{2 \tau q /(q-2)}\left(\mathbb{R}^{n}\right)$ implies that $u \in L^{\tau 2^{*}}\left(\mathbb{R}^{n}\right)$. If we set $q^{*}=2 q /(q-2)$, $\chi=2^{*} / q^{*}$ then $\tau \chi q^{*}=\tau 2^{*}$ and we have that

$$
\||u|\|_{\tau \lambda q^{*}}^{\tau} \leqslant C \tau\||u|\|_{\tau q^{*}}^{\tau}
$$

that is

$$
\||u|\|_{\tau \chi q^{*}} \leqslant C^{1 / \tau} \tau^{1 / \tau}\||u|\|_{\tau q^{*}}
$$


Let $\tau=\chi^{m}, m=0,1, \ldots$, then we have

$$
\|u\|_{\chi^{N} q^{*}} \leqslant \prod_{m=0}^{N-1}\left(C \chi^{m}\right)^{-\chi^{m}}\|u\|_{q^{*}} \leqslant C^{\sigma} \chi^{\tau}\|\mid u\|_{q^{*}} \leqslant C\|u\|_{q^{*}}
$$

where

$$
\sigma=\sum_{m=0}^{N-1} \chi^{-m}, \quad \tau=\sum_{m=0}^{N-1} m \chi^{-m}
$$
and $C$ is independent of $N$ for $\sum_{m=0}^{\infty} \chi^{-m}, \sum_{m=0}^{\infty} m \chi^{-m}$ are all convergent. Letting
$N \rightarrow \infty$ in (4.19) we get

$$
\|u\|_{\infty} \leqslant C\|u\|_{q^{*}}<+\infty
$$

Thus $u \in L_{\infty} \cap E$.

Finally, we show that for any $\varphi \in L_{\infty} \cap E$, we have

$$
\left.\frac{d}{d t} I\left(\frac{u+t \varphi}{\|u+t \varphi\|_{p}}\right)\right|_{t=0}=0 .
$$

Note that we only need to show that

$$
\left.\frac{d}{d t} I\left(\frac{u+t \varphi}{\|u+t \varphi\|_{p}}\right)\right|_{t=0}
$$

exists for any $\varphi \in L_{\infty} \cap E$. (4.21) can be proved by using the same method for proving (4.1). In fact, similarly to (4.2), (4.3), (4.4) and (4.5) we may obtain

$$
\frac{1}{2} \leqslant\|u+t \varphi\|_{p} \leqslant M \quad \text { (for } t \text { small enough) }
$$

$$
\frac{d}{d t}\|u+t \varphi\|_{p \mid t=0}=\int_{\mathbb{R}^{n}}|u|^{p-2} u^{i} \varphi^{i} d x
$$

(4.24) $\left.\frac{1}{d t} \int_{\mathbb{R}^{n}} \frac{h(x)|u+t \varphi|^{2}}{\|u+t \varphi\|_{p}^{2}} d x\right|_{t=0}=2 \int_{\mathbb{R}^{n}} h(x) u^{i} \varphi^{i} d x$

$$
-2 \int_{\mathbb{R}^{n}} h(x)|u|^{2} d x \int_{\mathbb{R}^{n}}|u|^{p-2} u^{i} \varphi^{i} d x
$$

(4.25) $I\left(\frac{u+t \varphi}{\|u+t \varphi\|_{p}}\right)=\int_{\mathbb{R} n} a_{\alpha \beta}\left(x, \frac{u+t \varphi}{\|u+t \varphi\|_{p}}\right) \frac{D_{\alpha} u^{i} D_{\beta} u^{i}}{\|u+t \varphi\|_{p}^{2}} d x$

$$
+2 t \int_{\mathbb{R} n} a_{\alpha \beta}\left(x, \frac{u+t \varphi}{\|u+t \varphi\|_{p}}\right) \frac{D_{\alpha} u^{i} D_{\beta} \varphi^{i}}{\|u+t \varphi\|_{p}^{2}} d x
$$




$$
\begin{aligned}
& +\frac{t^{2}}{\|u+t \varphi\|_{p}^{2}} \int_{\mathbb{R}^{n}} a_{\alpha \beta}\left(x, \frac{u+t \varphi}{\|u+t \varphi\|_{p}}\right) D_{\alpha} \varphi^{i} D_{\beta} \varphi^{i} d x \\
& +\int_{\mathbb{R}^{n}} \frac{h(x)|u+t \varphi|^{2}}{\|u+t \varphi\|_{p}^{2}} d x \\
& \equiv J^{1}(t)+J^{2}(t)+J^{3}(t)+\int_{\mathbb{R}^{n}} \frac{h(x)|u+t \varphi|^{2}}{\|u+t \varphi\|_{p}^{2}} d x .
\end{aligned}
$$

Using that $\|u\|_{\infty} \leqslant C,\|\varphi\|_{\infty} \leqslant C$, (4.22) and (ii) of Section 2, and similarly to (4.6) and (4.7) we obtain that

$$
\left.\frac{d}{d t} J^{2}(t)\right|_{t=0}=2 \int_{\mathbb{R}^{n}} a_{\alpha \beta}(x, u) D_{\alpha} u^{i} D_{\beta} \varphi^{i} d x,\left.\quad \frac{d}{d t} J^{3}(t)\right|_{t=0}=0
$$

On the other hand, we have that

$$
\begin{aligned}
\left.\frac{d}{d t} J^{1}(t)\right|_{t=0}= & \lim _{t \rightarrow 0} \int_{\mathbb{R} n} \frac{1}{t}\left[a_{\alpha \beta}\left(x, \frac{u+t \varphi}{\|u+t \varphi\|_{p}}\right)\|u+t \varphi\|_{p}^{-2}-a_{\alpha \beta}(x, u)\right] \\
= & \lim _{t \rightarrow 0} \int_{\mathbb{R}^{n}} \frac{1}{t}\left[u^{i} D_{\beta} u^{i} d x\right. \\
& \|u+t \varphi\|_{p}^{-2} D_{\alpha} u^{i} D_{\beta} u^{i} d x \\
& +\lim _{t \rightarrow 0} \frac{1}{t}\left(\|u+t \varphi\|_{p}^{-2}-1\right) \int_{\mathbb{R}^{n}} a_{\alpha \beta}(x, u) D_{\alpha} u^{i} D_{\beta} u^{i} d x \\
= & \lim _{t \rightarrow 0} J^{4}(t)+\lim _{t \rightarrow 0} J^{5}(t) .
\end{aligned}
$$

By (4.23) and similarly to (4.8) we obtain that

$$
\lim _{t \rightarrow 0} J^{5}(t)=-2 \int_{\mathbb{R}^{n}}|u|^{p-2} u^{i} \varphi^{i} d x \int_{\mathbb{R}^{n}} a_{\alpha \beta}(x, u) D_{\alpha} u^{i} D_{\beta} u^{i} d x .
$$

Using the mean value theorem we have that

$$
\begin{aligned}
\lim _{t \rightarrow 0} J^{4}(t)=\lim _{t \rightarrow 0} \int_{\mathbb{R}^{n}} D_{u^{j}} a_{\alpha \beta}\left(x, \frac{u+t^{\prime} \varphi}{\left\|u+t^{\prime} \varphi\right\|_{p}}\right) & \\
& {\left[\frac{\varphi^{j}}{\left\|u+t^{\prime} \varphi\right\|_{p}}-\left.\frac{u^{j}+t^{\prime} \varphi^{j}}{\left\|u+t^{\prime} \varphi\right\|_{p}^{2}} \frac{d}{d t}\|u+t \varphi\|_{p}\right|_{t=t^{\prime}}\right] } \\
& \|u+t \varphi\|_{p}^{-2} D_{\alpha} u^{i} D_{\beta} u^{i} d x
\end{aligned}
$$




$$
\begin{gathered}
=\lim _{t \rightarrow 0} \int_{\mathbb{R}^{n}} D_{u^{j}} a_{\alpha \beta}\left(x, \frac{u+t^{\prime} \varphi}{\left\|u+t^{\prime} \varphi\right\|_{p}}\right) \frac{\varphi^{j}}{\left\|u+t^{\prime} \varphi\right\|_{p}} \\
\|u+t \varphi\|_{p}^{-2} D_{\alpha} u^{i} D_{\beta} u^{i} d x \\
-\lim _{t \rightarrow 0} \int_{\mathbb{R}^{n}} D_{u j} a_{\alpha \beta}\left(x, \frac{u+t^{\prime} \varphi}{\left\|u+t^{\prime} \varphi\right\|_{p}}\right) \frac{u^{j}+t^{\prime} \varphi^{j}}{\left\|u+t^{\prime} \varphi\right\|_{p}^{2}} \\
\left.\|u+t \varphi\|_{p}^{-2} D_{\alpha} u^{i} D_{\beta} u^{i} \frac{d}{d t}\|u+t \varphi\|_{p}\right|_{t=t^{\prime}} d x \\
=\lim _{t \rightarrow 0} J^{6}(t)-\lim _{t \rightarrow 0} J^{7}(t),
\end{gathered}
$$

where $0<t^{\prime}(x)<t$. By (2.7) and (4.22) we see that

$$
\begin{gathered}
\left|D_{u^{j}} a_{\alpha \beta}\left(x, \frac{u+t^{\prime} \varphi}{\left\|u+t^{\prime} \varphi\right\|_{p}}\right) \frac{\varphi^{j}}{\left\|u+t^{\prime} \varphi\right\|_{p}}\|u+t \varphi\|_{p}^{-2} D_{\alpha} u^{i} D_{\beta} u^{i}\right| \\
\leqslant C \eta\left(\frac{|u|+t^{\prime}|\varphi|}{\left\|u+t^{\prime} \varphi\right\|_{p}}\right)\|u+t \varphi\|_{p}^{-2}|D u|^{2} \\
\leqslant C|D u|^{2} \in L^{1}\left(\mathbb{R}^{n}\right) .
\end{gathered}
$$

So, by the Dominated Convergence Theorem we have that

$$
\lim _{t \rightarrow 0} J^{6}(t)=\int_{\mathbb{R}^{n}} \varphi^{j} D_{u^{j}} a_{\alpha \beta}(x, u) D_{\alpha} u^{i} D_{\beta} u^{i} d x .
$$

Similarly to (4.11), we have that

$$
\lim _{t \rightarrow 0} J^{7}(t)=\int_{\mathbb{R} n}|u|^{p-2} u^{i} \varphi^{i} d x \int_{\mathbb{R} n} u^{j} D_{u^{j}} a_{\alpha \beta}(x, u) D_{\alpha} u^{i} D_{\beta} u^{i} d x
$$

Combining (4.24)-(4.27) we have that

$$
\begin{aligned}
0= & 2 \int_{\mathbb{R}^{n}} a_{\alpha \beta}(x, u) D_{\alpha} u^{i} D_{\beta} \varphi^{i} d x+\int_{\mathbb{R}^{n}} \varphi^{j} D_{u^{j}} a_{\alpha \beta}(x, u) D_{\alpha} u^{i} D_{\beta} u^{i} d x \\
& -\int_{\mathbb{R}^{n}}|u|^{p-2} u^{i} \varphi^{i} d x \int_{\mathbb{R}^{n}} u^{j} D_{u^{j}} a_{\alpha \beta}(x, u) D_{\alpha} u^{i} D_{\beta} u^{i} d x \\
& -2 \int_{\mathbb{R}^{n}}|u|^{p-2} u^{i} \varphi^{i} d x \int_{\mathbb{R}^{n}} a_{\alpha \beta}(x, u) D_{\alpha} u^{i} D_{\beta} u^{i} d x \\
& +2 \int_{\mathbb{R}^{n}} h(x) u^{i} \varphi^{i} d x-2 \int_{\mathbb{R}^{n}} h(x)|u|^{2} d x \int_{\mathbb{R}^{n}}|u|^{p-2} u^{i} \varphi^{i} d x
\end{aligned}
$$

which implies that

$$
\begin{aligned}
\int_{\mathbb{R}^{n}} a_{\alpha \beta}(x, u) D_{\alpha} u^{i} D_{\beta} \varphi^{i} d x+ & \frac{1}{2} \int_{\mathbb{R}^{n}} \varphi^{j} D_{u^{j}} a_{\alpha \beta}(x, u) D_{\alpha} u^{i} D_{\beta} u^{i} d x \\
& +\int_{\mathbb{R}^{n}} h(x) u^{i} \varphi^{i} d x=\lambda \int_{\mathbb{R}^{n}}|u|^{p-2} u^{i} \varphi^{i} d x
\end{aligned}
$$


for every $\varphi \in L_{\infty} \cap E$ where

$$
\lambda=\int_{\mathbb{R}^{n}}\left[a_{\alpha \beta}(x, u) D_{\alpha} u^{i} D_{\beta} u^{i}+\frac{1}{2} u^{j} D_{u^{j}} a_{\alpha \beta}(x, u) D_{\alpha} u^{i} D_{\beta} u^{i}+h(x)|u|^{2}\right] d x
$$

i.e. $u$ is a weak solution of (1.1) with $\|u\|_{\infty}<\infty$ and Theorem 2.3 is completely proved.

\section{References}

[1] $\mathrm{Ma} \mathrm{Li}$. On the positive solutions of quasilinear elliptic eigenvalue problem with limiting exponent (preprint).

[2] Shen Yiao-tian Eigenvalue problems of quasilinear elliptic systems (preprint).

[3] Giaquinta, M. Multiple integrals in the calculus of variations and nonlinear elliptic systems. Princeton University Press, 1983.

[4] P. L. Lions. The concentration-compactness principle in the calculus of variations. The locally compact case, Part 1 Ann. I.H.P. Anal. non linéaire, 1 (1984), 109-145.

[5] P.L. Lions. The concentration-compactness principle in the calculus of variations. The locally compact case, Part. 2 Ann. I.H.P. Anal. non linéaire, 1 (1984), 223-283.

[6] Yosida, K. Funcional Analysis. Springer-Verlag, 1978.

Li Gongbao

Wuhan Institute of Mathematical Sciences

Academia Sinica

P.O. Box 30 Wuhan

430071 P.R. of CHINA 